Network Working Group

Request for Comments: 4158

Category: Informational
M. Cooper

Orion Security Solutions

Y. Dzambasow

A\&N Associates

P. Hesse

Gemini Security Solutions

S. Joseph

Van Dyke Technologies

R. Nicholas

BAE Systems

September 2005

\title{
Internet X.509 Public Key Infrastructure: Certification Path Building
}

Status of This Memo

This memo provides information for the Internet community. It does not specify an Internet standard of any kind. Distribution of this memo is unlimited.

Copyright Notice

Copyright (C) The Internet Society (2005).

Abstract

This document provides guidance and recommendations to developers building X.509 public-key certification paths within their applications. By following the guidance and recommendations defined in this document, an application developer is more likely to develop a robust $\mathrm{X} .509$ certificate-enabled application that can build valid certification paths across a wide range of PKI environments.

Table of Contents

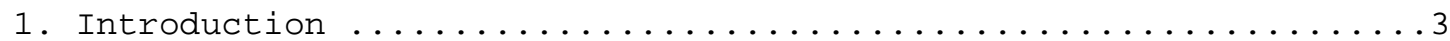

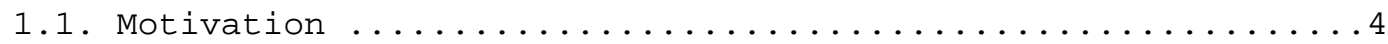

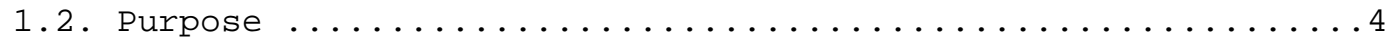

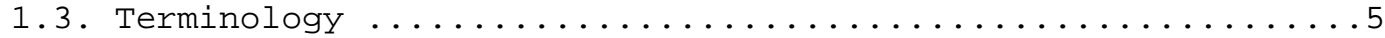

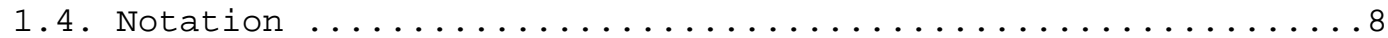

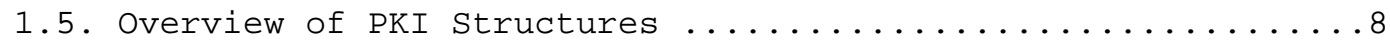
1.5.1. Hierarchical structures ..................

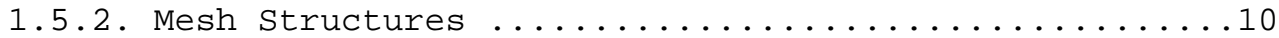

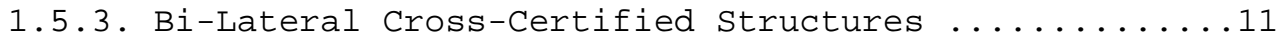

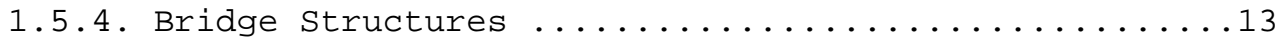

1.6. Bridge Structures and Certification Path Processing ......14 


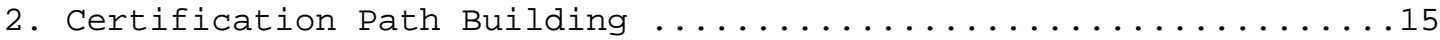

2.1. Introduction to Certification Path Building ..........15

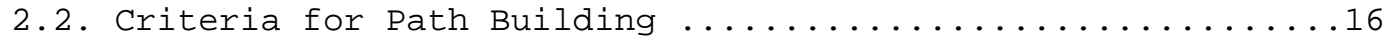

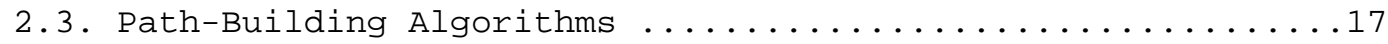

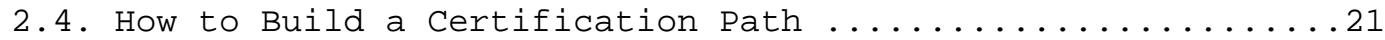

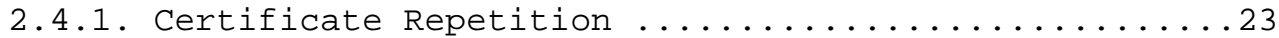

2.4.2. Introduction to Path-Building Optimization .......24

2.5. Building Certification Paths for Revocation Signer

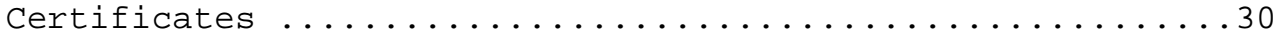

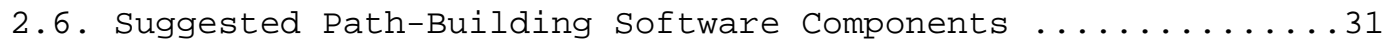

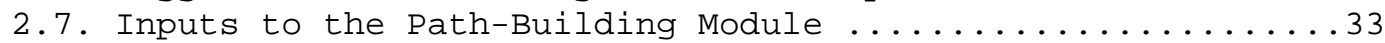

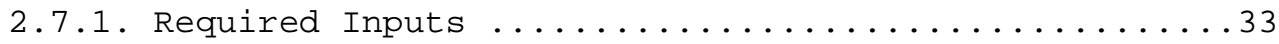

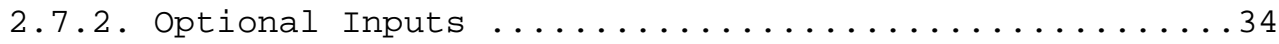

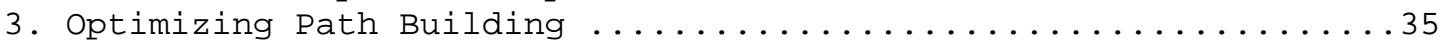

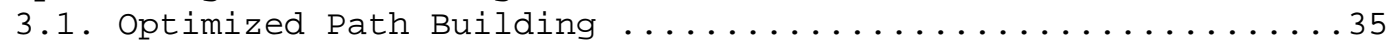

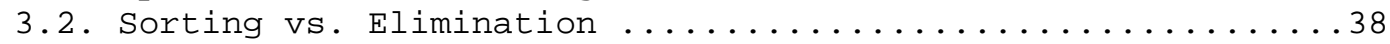

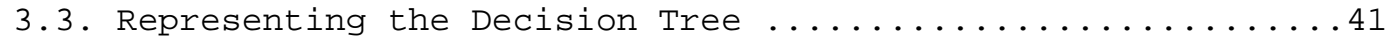

3.3.1. Node Representation for CA Entities ...........41

3.3.2. Using Nodes to Iterate Over All Paths ..........42

3.4. Implementing Path-Building Optimization ...........45

3.5. Selected Methods for Sorting Certificates ...........46

3.5.1. basicConstraints Is Present and CA Equals True ....47

3.5.2. Recognized Signature Algorithms .............48

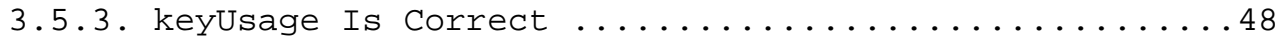

3.5.4. Time (T) Falls within the Certificate Validity ....48

3.5.5. Certificate Was Previously Validated ...........49

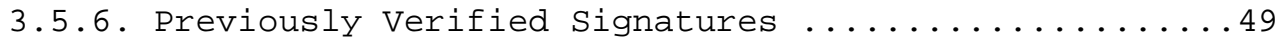

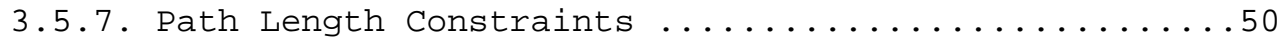

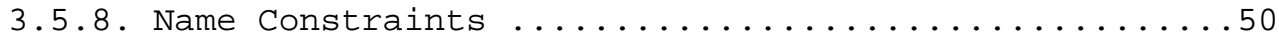

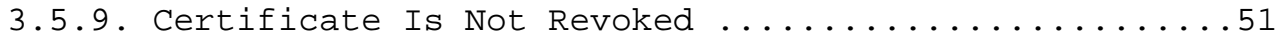

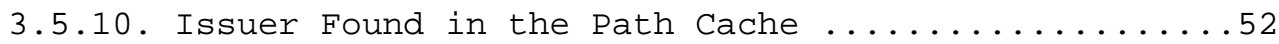

3.5.11. Issuer Found in the Application Protocol .......52

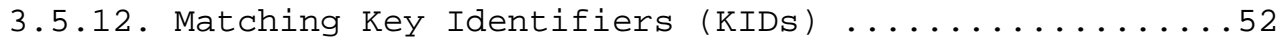

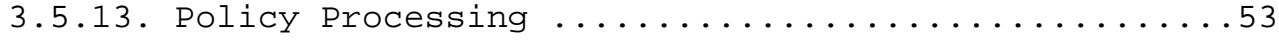

3.5.14. Policies Intersect the Sought Policy set .......54

3.5.15. Endpoint Distinguished Name (DN) Matching ......55

3.5.16. Relative Distinguished Name (RDN) Matching ......55

3.5.17. Certificates are Retrieved from

cACertificate Directory Attribute ............56

3.5.18. Consistent Public Key and Signature Algorithms ...56

3.5.19. Similar Issuer and Subject Names ............57

3.5.20. Certificates in the Certification Cache ........57

3.5.21. Current CRL Found in Local Cache ...............58

3.6. Certificate Sorting Methods for Revocation Signer

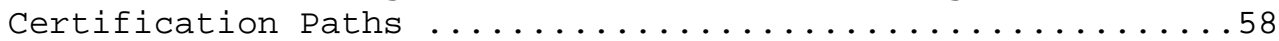

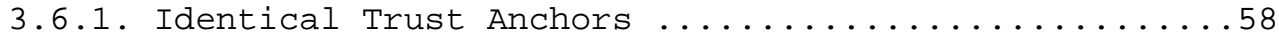

3.6.2. Endpoint Distinguished Name (DN) Matching .......59

3.6.3. Relative Distinguished Name (RDN) Matching ......59 
3.6.4. Identical Intermediate Names ..............60

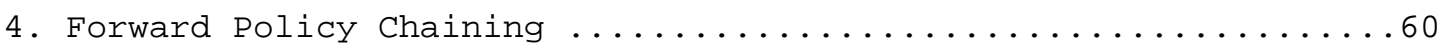

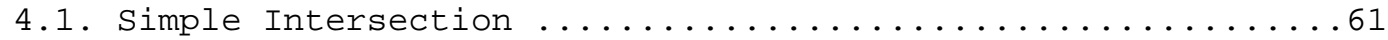

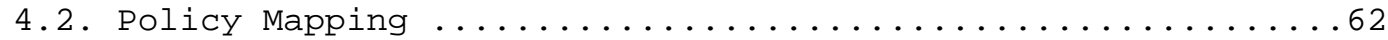

4.3. Assigning Scores for Forward Policy Chaining ........663

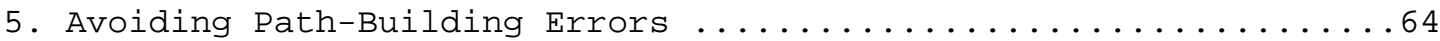

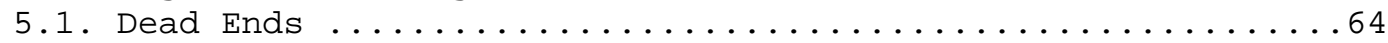

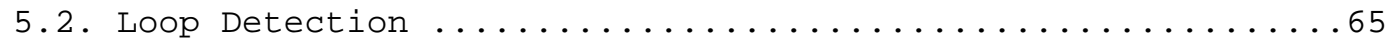

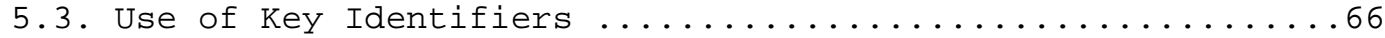

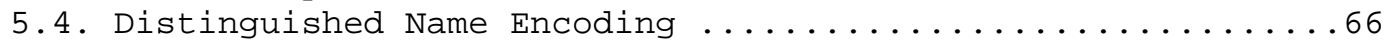

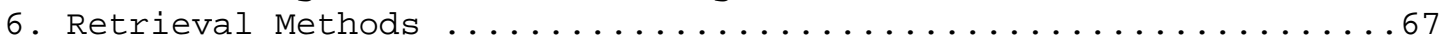

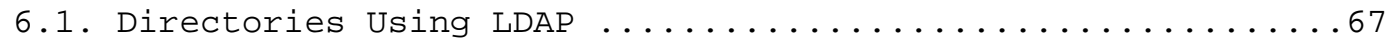

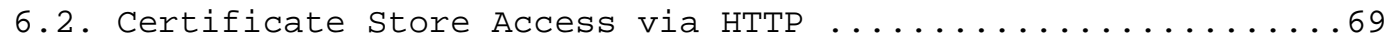

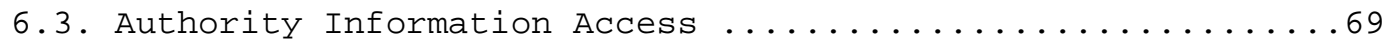

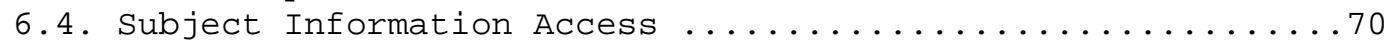

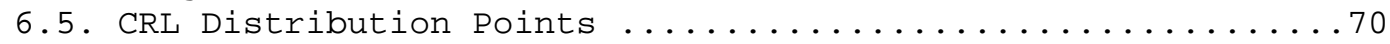

6.6. Data Obtained via Application Protocol ...............71

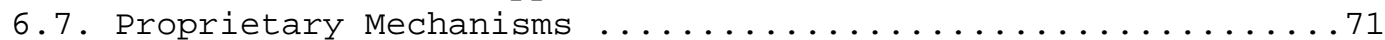

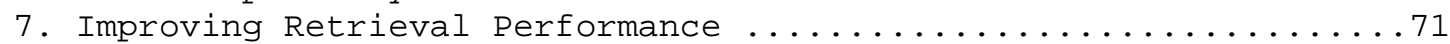

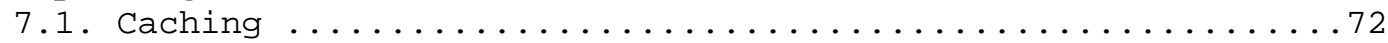

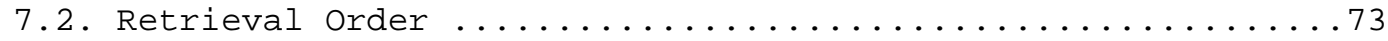

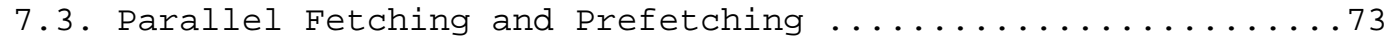

8. Security Considerations ............................ 74

8.1. General Considerations for Building a Certification Path ..74

8.2. Specific Considerations for Building Revocation

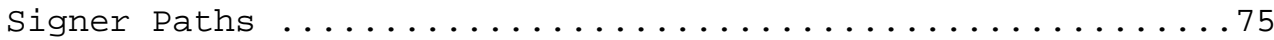

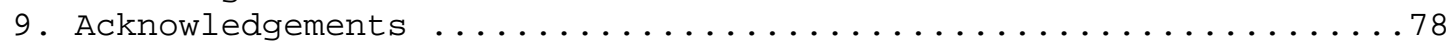

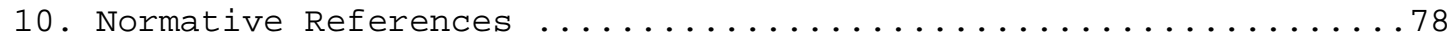

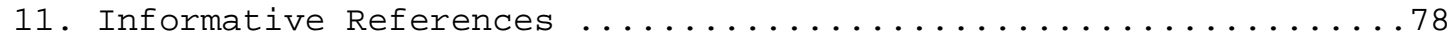

\section{Introduction}

[X.509] public key certificates have become an accepted method for securely binding the identity of an individual or device to a public key, in order to support public key cryptographic operations such as digital signature verification and public key-based encryption. However, prior to using the public key contained in a certificate, an application first has to determine the authenticity of that certificate, and specifically, the validity of all the certificates leading to a trusted public key, called a trust anchor. Through validating this certification path, the assertion of the binding made between the identity and the public key in each of the certificates can be traced back to a single trust anchor.

The process by which an application determines this authenticity of a certificate is called certification path processing. Certification path processing establishes a chain of trust between a trust anchor and a certificate. This chain of trust is composed of a series of 
certificates known as a certification path. A certification path begins with a certificate whose signature can be verified using a trust anchor and ends with the target certificate. Path processing entails building and validating the certification path to determine whether a target certificate is appropriate for use in a particular application context. See Section 3.2 of [RFC3280] for more information on certification paths and trust.

\subsection{Motivation}

Many other documents (such as [RFC3280]) cover certification path validation requirements and procedures in detail but do not discuss certification path building because the means used to find the path does not affect its validation. This document therefore is an effort to provide useful guidance for developers of certification pathbuilding implementations.

Additionally, the need to develop complex certification paths is increasing. Many PKIs are now using complex structures (see section 1.5) rather than simple hierarchies. Additionally, some enterprises are gradually moving away from trust lists filled with many trust anchors, and toward an infrastructure with one trust anchor and many cross-certified relationships. This document provides helpful information for developing certification paths in these more complicated situations.

\subsection{Purpose}

This document provides information and guidance for certification path building. There are no requirements or protocol specifications in this document. This document provides many options for performing certification path building, as opposed to just one particular way. This document draws upon the authors' experiences with existing complex certification paths to offer insights and recommendations to developers who are integrating support for [X.509] certificates into their applications.

In addition, this document suggests using an effective general approach to path building that involves a depth first tree traversal. While the authors believe this approach offers the balance of simplicity in design with very effective and infrastructure-neutral path-building capabilities, the algorithm is no more than a suggested approach. Other approaches (e.g., breadth first tree traversals) exist and may be shown to be more effective under certain conditions. Certification path validation is described in detail in both [X.509] and $[\mathrm{RFC} 3280]$ and is not repeated in this document. 
This document does not provide guidance for building the certification path from an end entity certificate to a proxy certificate as described in [RFC3820].

\subsection{Terminology}

Terms used throughout this document will be used in the following ways :

Building in the Forward direction: The process of building a certification path from the target certificate to a trust anchor. 'Forward' is the former name of the crosscertificatepair element ' issuedToThisCA'.

Building in the Reverse direction: The process of building a certification path from a trust anchor to the target certificate. 'Reverse' is the former name of the crosscertificatepair element ' issuedByThisCA'.

Certificate: A digital binding that cannot be counterfeited between a named entity and a public key.

Certificate Graph: A graph that represents the entire PKI (or all cross-certified PKIs) in which all named entities are viewed as nodes and all certificates are viewed as arcs between nodes.

Certificate Processing system: An application or device that performs the functions of certification path building and certification path validation.

Certification Authority (CA): An entity that issues and manages certificates.

Certification Path: An ordered list of certificates starting with a certificate signed by a trust anchor and ending with the target certificate.

Certification Path Building: The process used to assemble the certification path between the trust anchor and the target certificate.

Certification Path Validation: The process that verifies the binding between the subject and the subject-public-key defined in the target certificate, using a trust anchor and set of known constraints. 
Certificate Revocation List (CRL): A signed, time stamped list identifying a set of certificates that are no longer considered valid by the certificate issuer.

CRL Signer Certificate: The specific certificate that may be used for verifying the signature on a CRL issued by, or on behalf of, a specific CA.

Cross-Certificate: A certificate issued by one CA to another CA for the purpose of establishing a trust relationship between the two CAs .

Cross-Certification: The act of issuing cross-certificates.

Decision Tree: When the path-building software has multiple certificates to choose from, and must make a decision, the collection of possible choices is called a decision tree.

Directory: Generally used to refer an LDAP accessible repository for certificates and PKI information. The term may also be used generically to refer to any certificate storing repository.

End Entity: The holder of a private key and corresponding certificate, whose identity is defined as the subject of the certificate. Human end entities are often called "subscribers".

Is-revocation-signer indicator: A boolean flag furnished to the path-building software. If set, this indicates that the target certificate is a Revocation Signer certificate for a specific CA. For example, if building a certification path for an indirect CRL Signer certificate, this flag would be set.

Local PKI: The set of PKI components and data (certificates, directories, CRLs, etc.) that are created and used by the certificate using organization. In general, this concept refers to the components that are in close proximity to the certificate using application. The assumption is that the local data is more easily accessible and/or inexpensive to retrieve than non-local PKI data.

Local Realm: See Local PKI.

Node (in a certificate graph): The collection of certificates having identical subject distinguished names.

Online Certificate Status Protocol (OCSP): An Internet protocol used by a client to obtain the revocation status of a certificate from a server. 
OCSP Response Signer Certificate: The specific certificate that may be used for verifying the signature on an OCSP response. This response may be provided by the $\mathrm{CA}$, on behalf of the $\mathrm{CA}$, or by a different signer as determined by the Relying Party's local policy.

Public Key Infrastructure (PKI): The set of hardware, software, personnel, policy, and procedures used by a CA to issue and manage certificates.

Relying Party (RP): An application or entity that processes certificates for the purpose of 1) verifying a digital signature, 2) authenticating another entity, or 3) establishing confidential communications.

Revocation Signer Certificate: Refers collectively to either a CRL Signer Certificate or OCSP Response Signer Certificate.

Target Certificate: The certificate that is to be validated by a Relying Party. It is the "Certificate targeted for validation". Although frequently this is the End Entity or a leaf node in the PKI structure, this could also be a CA certificate if a CA certificate is being validated. (e.g., This could be for the purpose of building and validating a certification path for the signer of a CRL.)

Trust (of public keys): In the scope of this document, a public key is considered trustworthy if the certificate containing the public key can be validated according to the procedures in [RFC3280].

Trust List: A list of trust anchors.

Trust Anchor: The combination of a trusted public key and the name of the entity to which the corresponding private key belongs.

Trust Anchor Certificate: A self-signed certificate for a trust anchor that is used in certification path processing.

User: An individual that is using a certificate processing system. This document refers to some cases in which users may or may not be prompted with information or requests, depending upon the implementation of the certificate processing system. 


\subsection{Notation}

This document makes use of a few common notations that are used in the diagrams and examples.

The first is the arrow symbol $(->)$ which represents the issuance of a certificate from one entity to another. For example, if entity $\mathrm{H}$ were to issue a certificate to entity $\mathrm{K}$, this is denoted as $\mathrm{H}->\mathrm{K}$.

Sometimes it is necessary to specify the subject and issuer of a given certificate. If entity $\mathrm{H}$ were to issue a certificate to entity $K$ this can be denoted as $K(H)$.

These notations can be combined to denote complicated certification paths such as $C(D)->B(C)->A(B)$.

\subsection{Overview of PKI Structures}

When verifying [X.509] public key certificates, often the application performing the verification has no knowledge of the underlying Public Key Infrastructure (PKI) that issued the certificate. PKI structures can range from very simple, hierarchical structures to complex structures such as mesh architectures involving multiple bridges (see Section 1.5.4). These structures define the types of certification paths that might be built and validated by an application [MINHPKIS] . This section describes four common PKI structures.

\subsubsection{Hierarchical structures}

A hierarchical PKI, depicted in Figure 1, is one in which all of the end entities and relying parties use a single "Root CA" as their trust anchor. If the hierarchy has multiple levels, the Root CA certifies the public keys of intermediate CAs (also known as subordinate CAs). These CAs then certify end entities' (subscribers') public keys or may, in a large PKI, certify other CAs. In this architecture, certificates are issued in only one direction, and a CA never certifies another CA "superior" to itself. Typically, only one superior CA certifies each CA. 


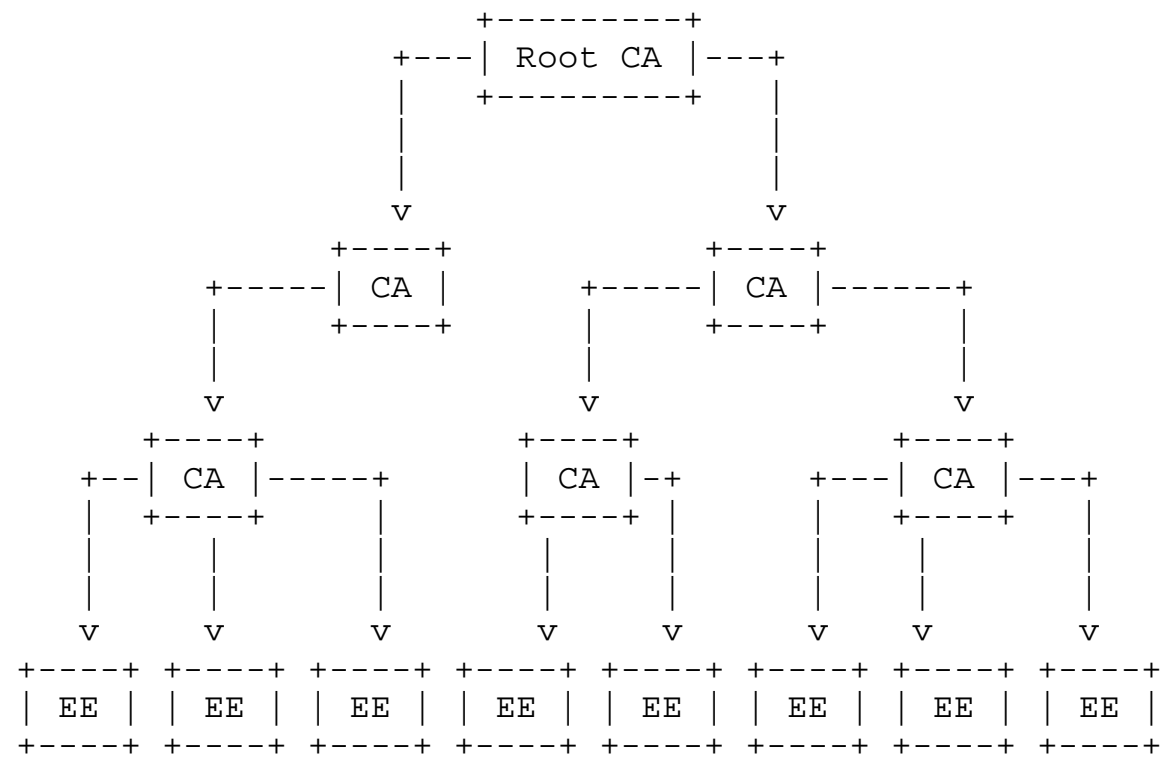

Figure 1 - Sample Hierarchical PKI

Certification path building in a hierarchical PKI is a straightforward process that simply requires the relying party to successively retrieve issuer certificates until a certificate that was issued by the trust anchor (the "Root CA" in Figure 1) is located.

A widely used variation on the single-rooted hierarchical PKI is the inclusion of multiple CAs as trust anchors. (See Figure 2.) Here, end entity certificates are validated using the same approach as with any hierarchical PKI. The difference is that a certificate will be accepted if it can be verified back to any of the set of trust anchors. Popular web browsers use this approach, and are shipped with trust lists containing dozens to more than one hundred CAs. While this approach simplifies the implementation of a limited form of certificate verification, it also may introduce certain security vulnerabilities. For example, the user may have little or no idea of the policies or operating practices of the various trust anchors, and may not be aware of which root was used to verify a given certificate. Additionally, the compromise of any trusted CA private key or the insertion of a rogue CA certificate to the trust list may compromise the entire system. Conversely, if the trust list is properly managed and kept to a reasonable size, it can be an efficient solution to building and validating certification paths. 


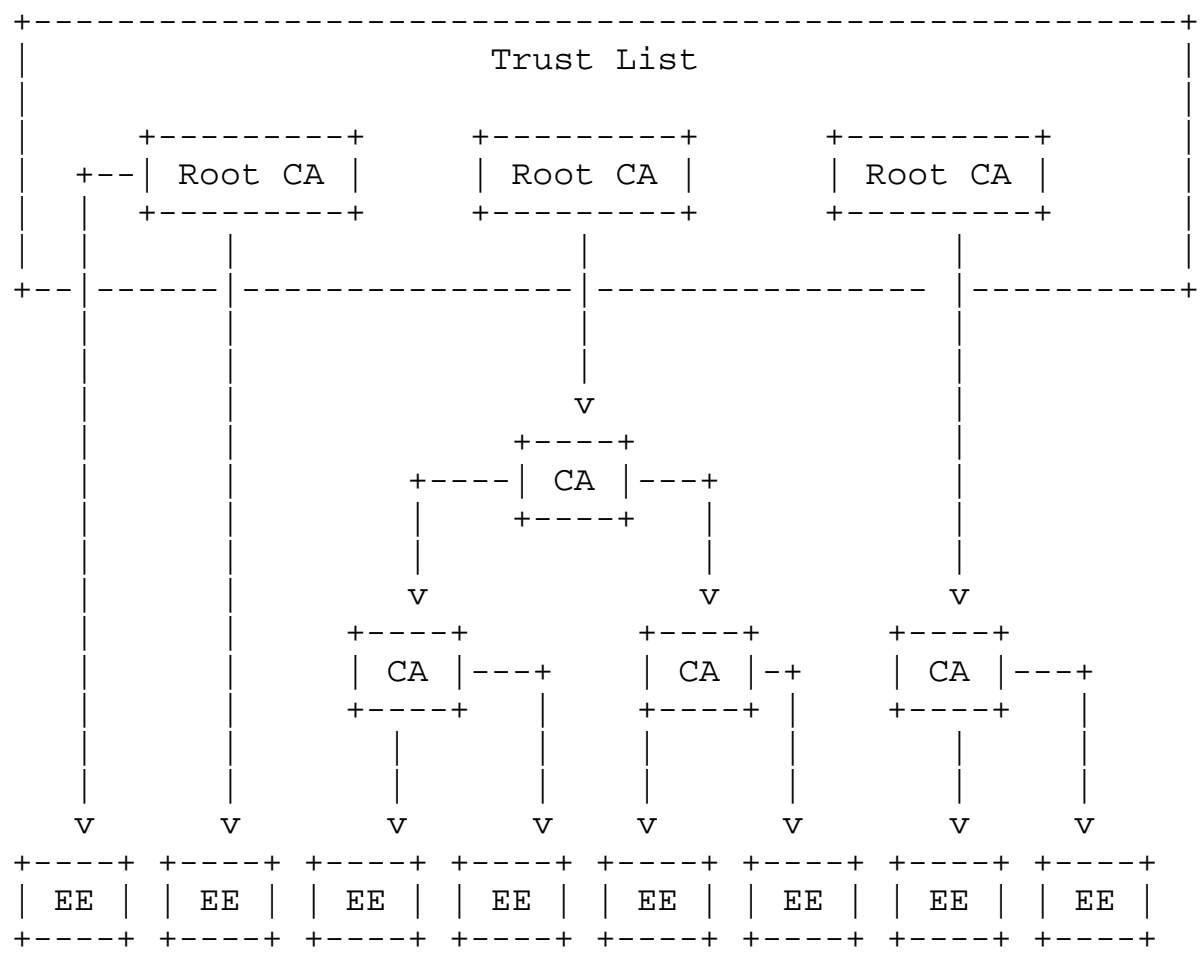

Figure 2 - Multi-Rooted Hierarchical PKI

\subsubsection{Mesh Structures}

In a typical mesh style PKI (depicted in Figure 3), each end entity trusts the CA that issued their own certificate(s). Thus, there is no 'Root CA' for the entire PKI. The CAs in this environment have peer relationships; they are neither superior nor subordinate to one another. In a mesh, CAs in the PKI cross-certify. That is, each CA issues a certificate to, and is issued a certificate by, peer CAs in the PKI. The figure depicts a mesh PKI that is fully cross-certified (sometimes called a full mesh). However, it is possible to architect and deploy a mesh PKI with a mixture of uni-directional and bidirectional cross-certifications (called a partial mesh). Partial meshes may also include CAs that are not cross-certified with other CAs in the mesh. 


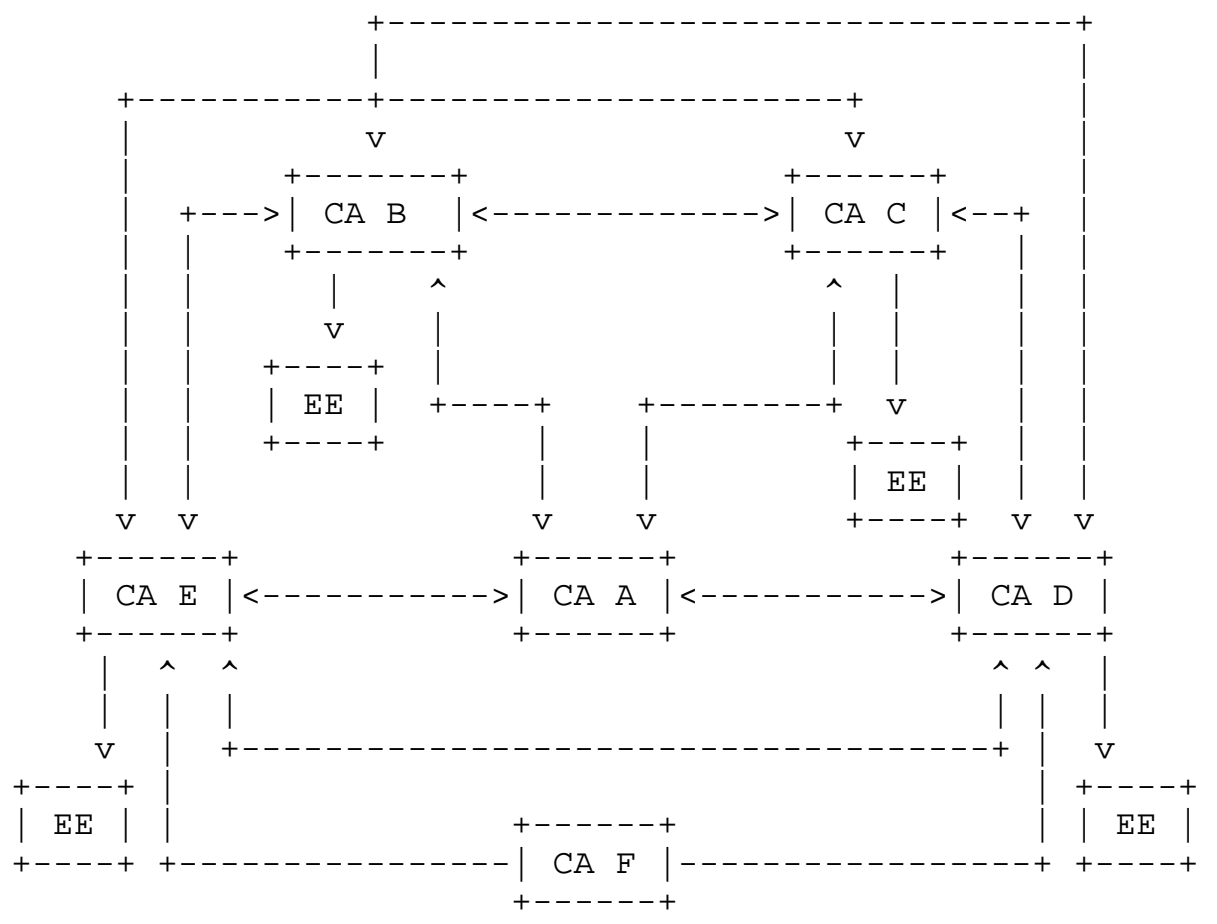

Figure 3 - Mesh PKI

Certification path building in a mesh PKI is more complex than in a hierarchical PKI due to the likely existence of multiple paths between a relying party's trust anchor and the certificate to be verified. These multiple paths increase the potential for creating "loops", "dead ends", or invalid paths while building the certification path between a trust anchor and a target certificate. In addition, in cases where no valid path exists, the total number of paths traversed by the path-building software in order to conclude "no path exists" can grow exceedingly large. For example, if ignoring everything except the structure of the graph, the Mesh PKI figure above has 22 non-self issued CA certificates and a total of 5,092,429 certification paths between CA F and the EE issued by CA D without repeating any certificates.

\subsubsection{Bi-Lateral Cross-Certified Structures}

PKIs can be connected via cross-certification to enable the relying parties of each to verify and accept certificates issued by the other PKI. If the PKIs are hierarchical, cross-certification will typically be accomplished by each Root CA issuing a certificate for the other PKI's Root CA. This results in a slightly more complex, 
but still essentially hierarchical environment. If the PKIs are mesh style, then a CA within each PKI is selected, more or less arbitrarily, to establish the cross-certification, effectively creating a larger mesh PKI. Figure 4 depicts a hybrid situation resulting from a hierarchical PKI cross-certifying with a mesh PKI.

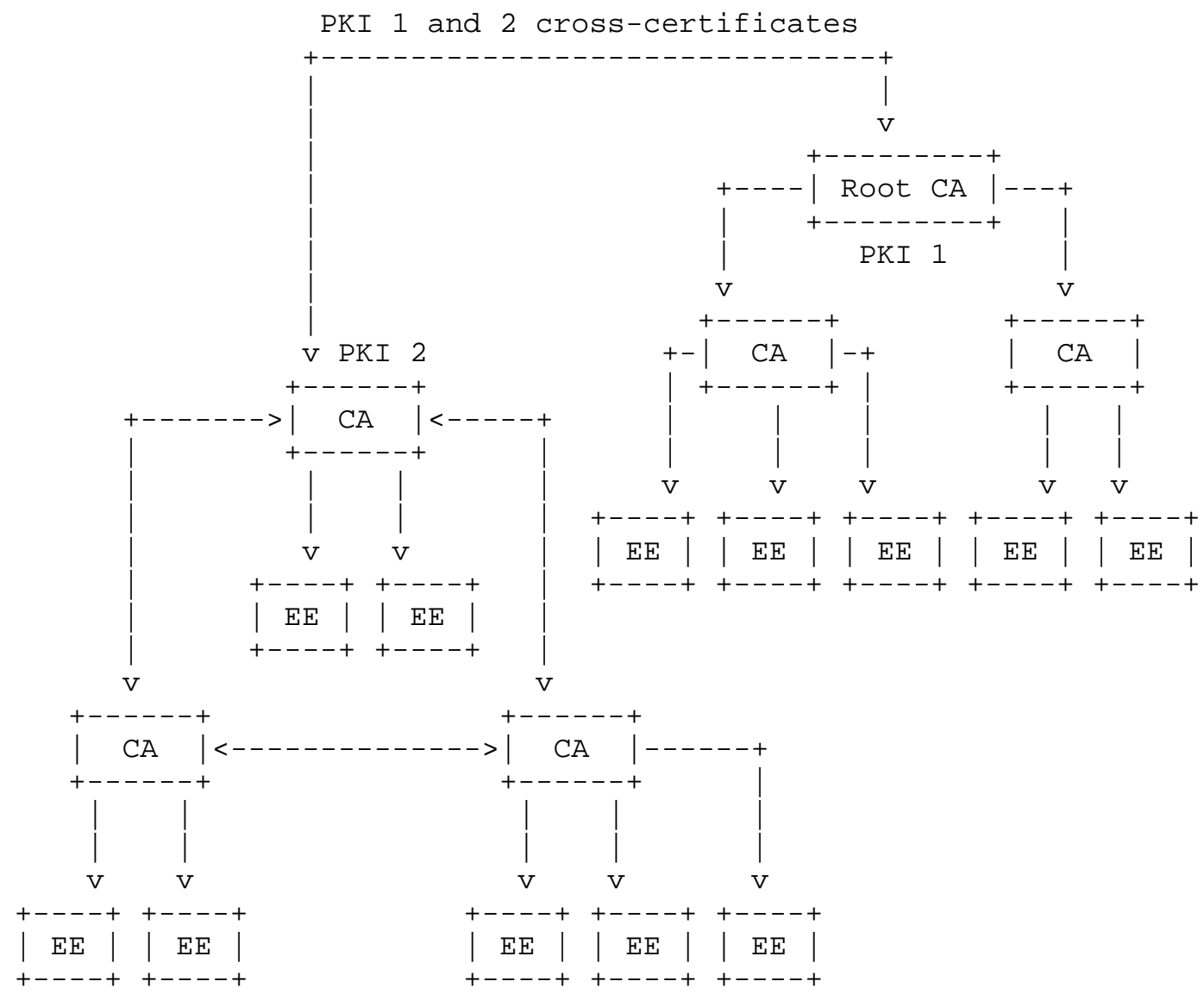

Figure 4 - Hybrid PKI

In current implementations, this situation creates a concern that the applications used under the hierarchical PKIs will not have path building capabilities robust enough to handle this more complex certificate graph. As the number of cross-certified PKIs grows, the number of the relationships between them grows exponentially. Two principal concerns about cross-certification are the creation of unintended certification paths through transitive trust, and the dilution of assurance when a high-assurance PKI with restrictive operating policies is cross-certified with a PKI with less 
restrictive policies. (Proper name constraints and certificate policies processing can help mitigate the problem of assurance dilution.)

\subsubsection{Bridge Structures}

Another approach to the interconnection of PKIs is the use of a "bridge" certification authority (BCA). A BCA is a nexus to establish trust paths among multiple PKIs. The BCA cross-certifies with one CA in each participating PKI. Each PKI only cross-certifies with one other CA (i.e., the BCA), and the BCA cross-certifies only once with each participating PKI. As a result, the number of cross certified relationships in the bridged environment grows linearly with the number of PKIs whereas the number of cross-certified relationships in mesh architectures grows exponentially. However, when connecting PKIs in this way, the number and variety of PKIs involved results in a non-hierarchical environment, such as the one as depicted in Figure 5. (Note: as discussed in Section 2.3, nonhierarchical PKIs can be considered hierarchical, depending upon perspective.) 


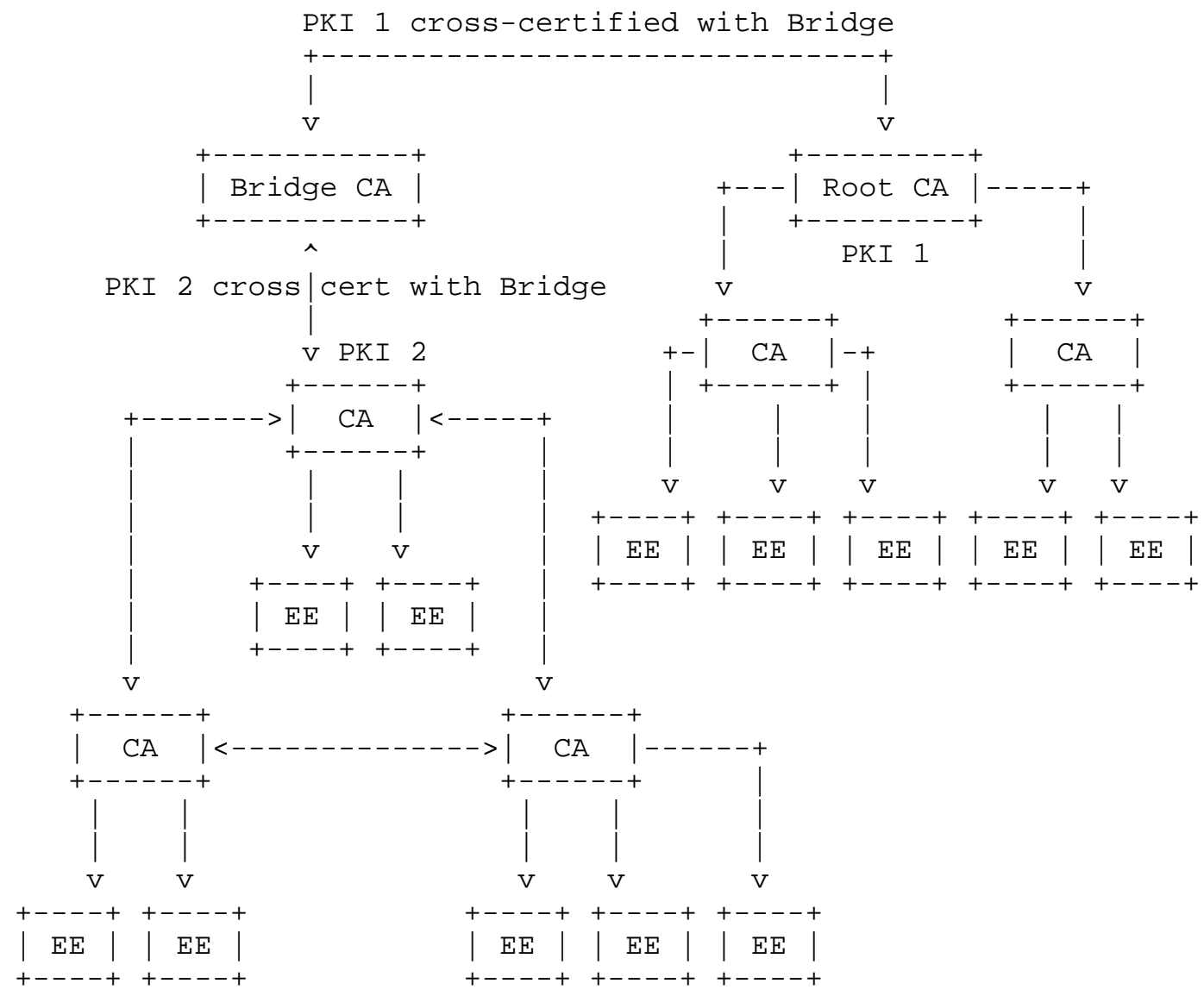

Figure 5 - Cross-Certification with a Bridge CA

\subsection{Bridge Structures and Certification Path Processing}

Developers building certificate-enabled applications intended for widespread use throughout various sectors are encouraged to consider supporting a Bridge PKI structure because implementation of certification path processing functions to support a Bridge PKI structure requires support of all the PKI structures (e.g., hierarchical, mesh, hybrid) which the Bridge may connect. An application that can successfully build valid certification paths in all Bridge PKIs will therefore have implemented all of the processing logic required to support the less complicated PKI structures. Thus, if an application fully supports the Bridge PKI structure, it can be deployed in any standards-compliant PKI environment and will perform the required certification path processing properly. 


\section{Certification Path Building}

Certification path building is the process by which the certificate processing system obtains the certification path between a trust anchor and the target certificate. Different implementations can build the certification path in different ways; therefore, it is not the intent of this document to recommend a single "best" way to perform this function. Rather, guidance is provided on the technical issues that surround the path-building process, and on the capabilities path-building implementations need in order to build certification paths successfully, irrespective of PKI structures.

\subsection{Introduction to Certification Path Building}

A certification path is an ordered list of certificates starting with a certificate that can be validated by one of the relying party's trust anchors, and ending with the certificate to be validated. (The certificate to be validated is referred to as the "target certificate" throughout this document.) Though not required, as a matter of convenience these trust anchors are typically stored in trust anchor certificates. The intermediate certificates that comprise the certification path may be retrieved by any means available to the validating application. These sources may include LDAP, HTTP, SQL, a local cache or certificate store, or as part of the security protocol itself as is common practice with signed S/MIME messages and SSL/TLS sessions.

Figure 6 shows an example of a certification path. In this figure, the horizontal arrows represent certificates, and the notation $B(A)$ signifies a certificate issued to B, signed by A.

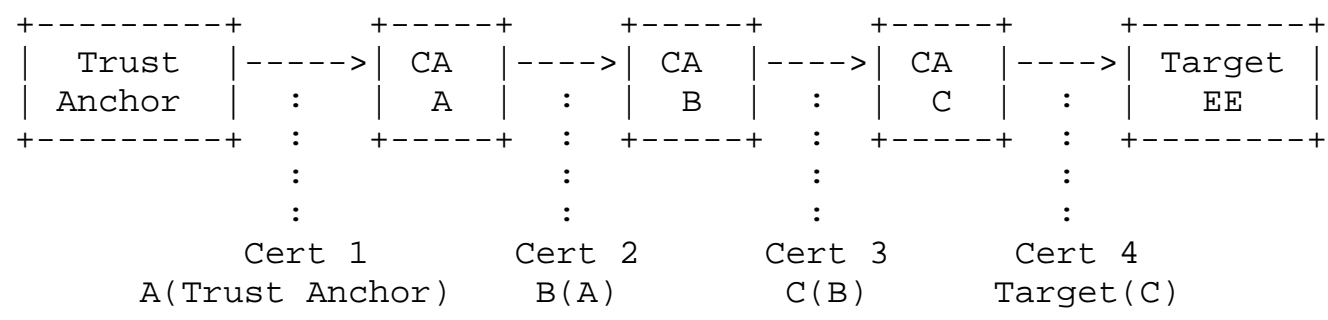

Figure 6 - Example Certification Path

Unlike certification path validation, certification path building is not addressed by the standards that define the semantics and structure of a PKI. This is because the validation of a certification path is unaffected by the method in which the certification path was built. However, the ability to build a valid certification path is of paramount importance for applications that 
rely on a PKI. Without valid certification paths, certificates cannot be validated according to [RFC3280] and therefore cannot be trusted. Thus, the ability to build a path is every bit as important as the ability to validate it properly.

There are many issues that can complicate the path-building process. For example, building a path through a cross-certified environment could require the path-building module to traverse multiple PKI domains spanning multiple directories, using multiple algorithms, and employing varying key lengths. A path-building client may also need to manage a number of trust anchors, partially populated directory entries (e.g., missing issuedToThisCA entries in the crosscertificatePair attribute), parsing of certain certificate extensions (e.g., authorityInformationAccess) and directory attributes (e.g., crosscertificatePair), and error handling such as loop detection.

In addition, a developer has to decide whether to build paths from a trust anchor (the reverse direction) to the target certificate or from the target certificate (the forward direction) to a trust anchor. Some implementations may even decide to use both. The choice a developer makes should be dependent on the environment and the underlying PKI for that environment. More information on making this choice can be found in section 2.3.

\subsection{Criteria for Path Building}

From this point forward, this document will be discussing specific algorithms and mechanisms to assist developers of certification path-building implementations. To provide justification for these mechanisms, it is important to denote what the authors considered the criteria for a path-building implementation.

Criterion 1: The implementation is able to find all possible paths, excepting paths containing repeated subject name/public key pairs. This means that all potentially valid certification paths between the trust anchor and the target certificate which may be valid paths can be built by the algorithm. As discussed in section 2.4.2, we recommend that subject names and public key pairs are not repeated in paths.

Criterion 2: The implementation is as efficient as possible. An efficient certification path-building implementation is defined to be one that builds paths that are more likely to validate following [RFC3280], before building paths that are not likely to validate, with the understanding that there is no way to account for all possible configurations and infrastructures. This criterion is intended to ensure implementations that can produce useful error 
information. If a particular path is entirely valid except for a single expired certificate, this is most likely the 'right' path. If other paths are developed that are invalid for multiple obscure reasons, this provides little useful information.

The algorithms and mechanisms discussed henceforth are chosen because the authors consider them to be good methods for meeting the above criteria.

\subsection{Path-Building Algorithms}

It is intuitive for people familiar with the Bridge CA concept or mesh type PKIs to view path building as traversing a complex graph. However, from the simplest viewpoint, writing a path-building module can be nothing more than traversal of a spanning tree, even in a very complex cross-certified environment. Complex environments as well as hierarchical PKIs can be represented as trees because certificates are not permitted to repeat in a path. If certificates could be repeated, loops can be formed such that the number of paths and number of certificates in a path both increase without bound (e.g., A issues to B, B issues to $C$, and C issues to A). Figure 7 below illustrates this concept from the trust anchor's perspective. 


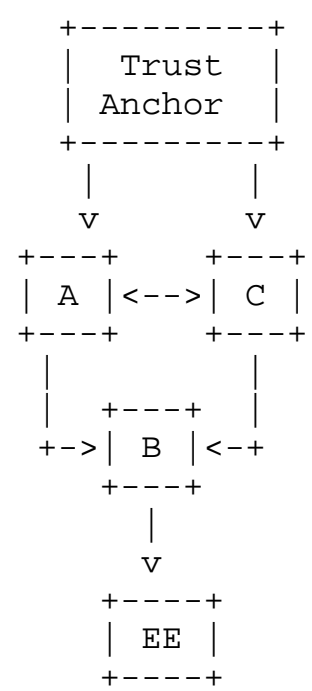

A certificate graph with bi-directional cross-cert. between CAs $A$ and $C$.

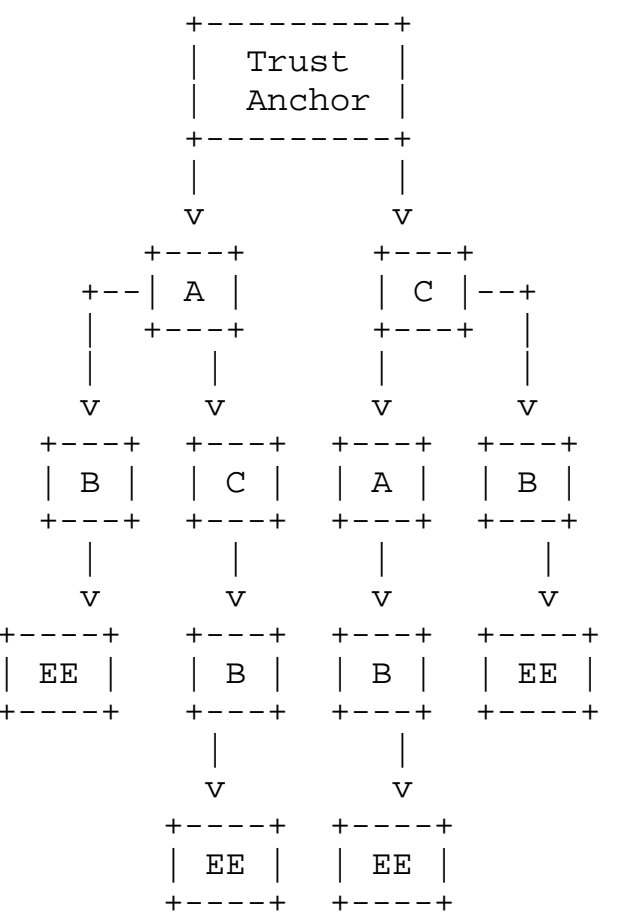

The same certificate graph rendered as a tree - the way path-building software could see it.

Figure 7 - Simple Certificate Graph - From Anchor Tree Depiction

When viewed from this perspective, all PKIs look like hierarchies emanating from the trust anchor. An infrastructure can be depicted in this way regardless of its complexity. In Figure 8, the same graph is depicted from the end entity (EE) (the target certificate in this example). It would appear this way if building in the forward (from EE or from target) direction. In this example, without knowing any particulars of the certificates, it appears at first that building from $\mathrm{EE}$ has a smaller decision tree than building from the trust anchor. While it is true that there are fewer nodes in the tree, it is not necessarily more efficient in this example. 


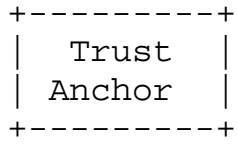

$\wedge$

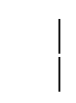

$\mid \begin{gathered}\mathrm{A} \\ +---+\end{gathered}$

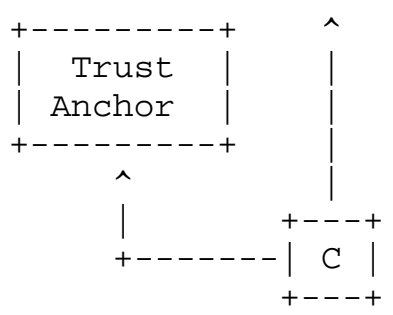

$\wedge$
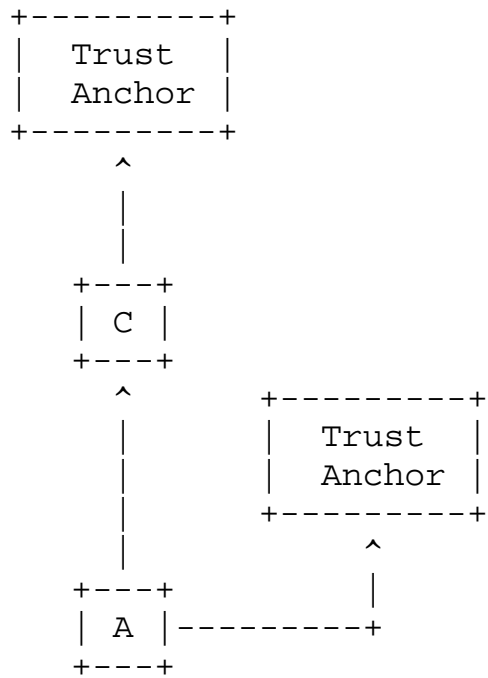

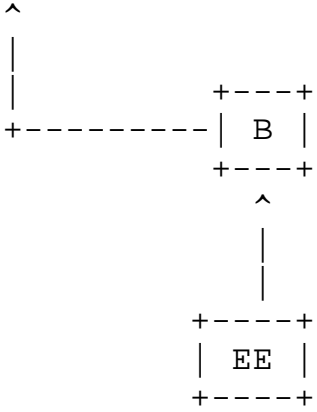

The same certificate graph rendered

as a tree but from the end entity rather than the trust anchor.

Figure 8 - Certificate Graph - From Target Certificate Depiction

Suppose a path-building algorithm performed no optimizations. That is, the algorithm is only capable of detecting that the current certificate in the tree was issued by the trust anchor, or that it issued the target certificate (EE). From the tree above, building from the target certificate will require going through two intermediate certificates before encountering a certificate issued by the trust anchor 100\% of the time (e.g., EE chains to B, which then chains to $C$, which is issued by the Trust Anchor). The path-building module would not chain $C$ to $A$ because it can recognize that $C$ has a certificate issued by the Trust Anchor (TA). 
On the other hand, in the first tree (Figure 7: from anchor depiction), there is a 50\% probability of building a path longer than needed (e.g., TA to A to C to B to EE rather than the shorter TA to A to $B$ to EE). However, even given our simplistic example, the pathbuilding software, when at $A$, could be designed to recognize that $B^{\prime} s$ subject distinguished name (DN) matches the issuer DN of the EE. Given this one optimization, the builder could prefer B to C. (B's subject DN matches that of the EE's issuer whereas $C^{\prime} s$ subject DN does not.) So, for this example, assuming the issuedByThisCA (reverse) and issuedToThisCA (forward) elements were fully populated in the directory and our path-building module implemented the aforementioned DN matching optimization method, path building from either the trust anchor or the target certificate could be made roughly equivalent. A list of possible optimization methods is provided later in this document.

A more complicated example is created when the path-building software encounters a situation when there are multiple certificates from which to choose while building a path. We refer to this as a large decision tree, or a situation with high fan-out. This might occur if an implementation has multiple trust anchors to choose from, and is building in the reverse (from trust anchor) direction. Or, it may occur in either direction if a Bridge CA is encountered. Large decision trees are the enemy of efficient path-building software. To combat this problem, implementations should make careful decisions about the path-building direction, and should utilize optimizations such as those discussed in section 3.1 when confronted with a large decision tree.

Irrespective of the path-building approach for any path-building algorithm, cases can be constructed that make the algorithm perform poorly. The following questions should help a developer decide from which direction to build certification paths for their application:

1) What is required to accommodate the local PKI environment and the PKI environments with which interoperability will be required?

a. If using a directory, is the directory [RFC2587] compliant (specifically, are the issuedToThisCA [forward] crosscertificates and/or the cACertificate attributes fully populated in the directory)? If yes, you are able to build in the forward direction.

b. If using a directory, does the directory contain all the issuedByThiscA (reverse) cross-certificates in the crossCertificatePair attribute, or, alternately, are all certificates issued from each CA available via some other means? If yes, it is possible to build in the reverse 
direction. Note: [RFC2587] does not require the issuedByThisCA (reverse) cross-certificates to be populated; if they are absent it will not be possible to build solely in the reverse direction.

c. Are all issuer certificates available via some means other than a directory (e.g., the authorityInformationAccess extension is present and populated in all certificates)? If yes, you are able to build in the forward direction.

2) How many trust anchors will the path-building and validation software be using?

a. Are there (or will there be) multiple trust anchors in the local PKI? If yes, forward path building may offer better performance.

b. Will the path-building and validation software need to place trust in trust anchors from PKIs that do not populate reverse cross-certificates for all intermediate CAs? If no, and the local PKI populates reverse cross-certificates, reverse path building is an option.

\subsection{How to Build a Certification Path}

As was discussed in the prior section, path building is essentially a tree traversal. It was easy to see how this is true in a simple example, but how about a more complicated one? Before taking a look at more a complicated scenario, it is worthwhile to address loops and what constitutes a loop in a certification path. [X.509] specifies that the same certificate may not repeat in a path. In a strict sense, this works well as it is not possible to create an endless loop without repeating one or more certificates in the path. However, this requirement fails to adequately address Bridged PKI environments. 


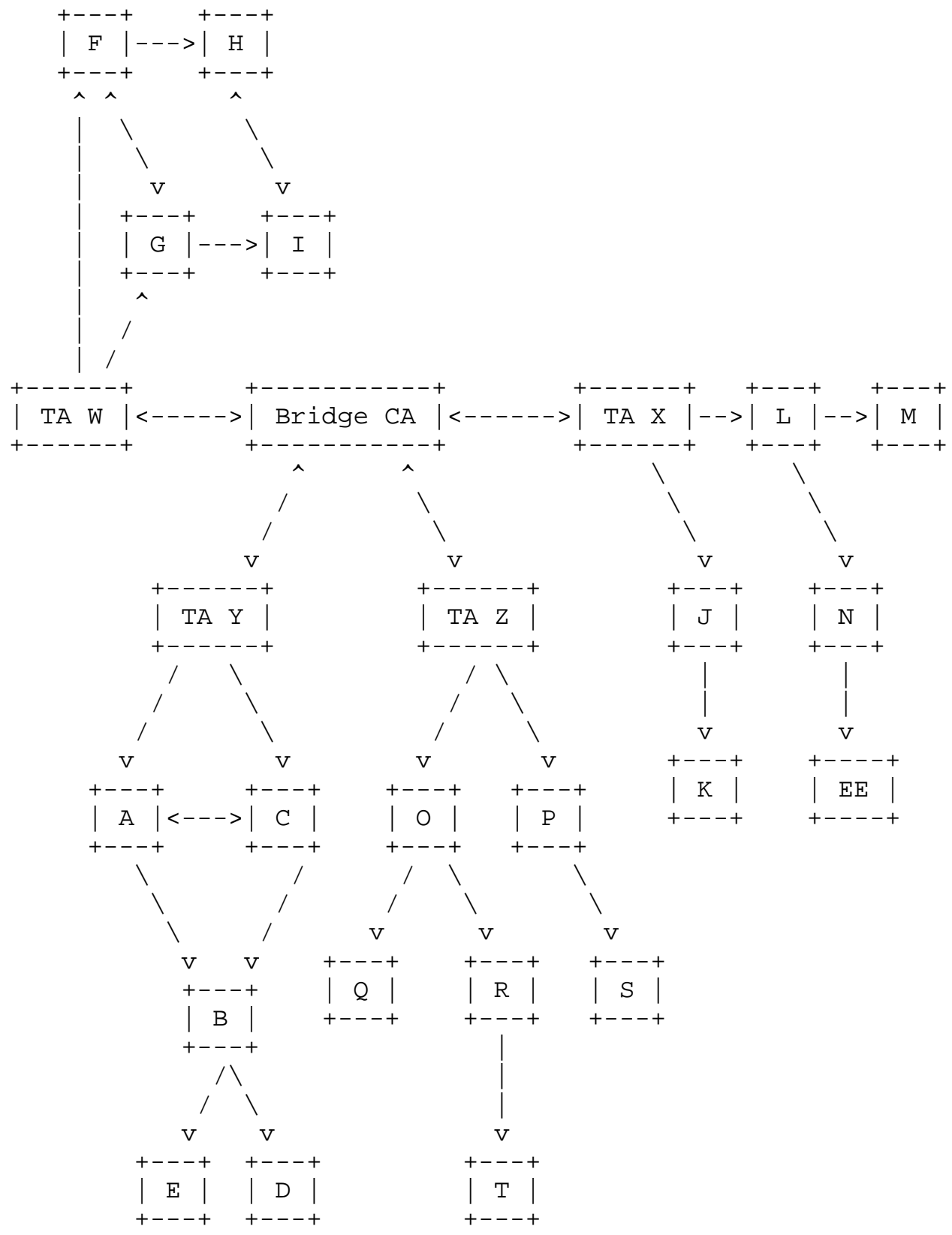

Figure 9 - Four Bridged PKIs 
Figure 9 depicts four root certification authorities cross-certified with a Bridge CA (BCA). While multiple trust anchors are shown in the Figure, our examples all consider TA $Z$ as the trust anchor. The other trust anchors serve different relying parties. By building certification paths through the BCA, trust can be extended across the four infrastructures. In Figure 9, the BCA has four certificates issued to it; one issued from each of the trust anchors in the graph. If stored in the BCA directory system, the four certificates issued to the BCA would be stored in the issuedToThisCA (forward) entry of four different crossCertificatePair structures. The BCA also has issued four certificates, one to each of the trust anchors. If stored in the BCA directory system, those certificates would be stored in the issuedByThisCA (reverse) entry of the same four crosscertificatePair structures. (Note that the cross-certificates are stored as matched pairs in the crosscertificatePair attribute. For example, a crosscertificatePair structure might contain both $A(B)$ and $B(A)$, but not contain $A(C)$ and $B(A)$.$) The four$ crosscertificatePair structures would then be stored in the BCA' $^{\prime}$ directory entry in the crosscertificatepair attribute.

\subsubsection{Certificate Repetition}

[X.509] requires that certificates are not repeated when building paths. For instance, from the figure above, do not build the path TA $\mathrm{Z}->\mathrm{BCA}->\mathrm{Y}->\mathrm{A}->\mathrm{C}->\mathrm{A}->\mathrm{C}->\mathrm{B}->\mathrm{D}$. Not only is the repetition unnecessary to build the path from $Z$ to $D$, but it also requires the reuse of $a$ certificate (the one issued from C to A), which makes the path noncompliant with [X.509].

What about the following path from TA Z to EE?

$$
\text { TA } \quad \mathrm{Z}->\mathrm{BCA}->\mathrm{Y}->\mathrm{BCA}->\mathrm{W}->\mathrm{BCA}->\mathrm{X}->\mathrm{L}->\mathrm{N}->\mathrm{EE}
$$

Unlike the first example, this path does not require a developer to repeat any certificates; therefore, it is compliant with [X.509]. Each of the BCA certificates is issued from a different source and is therefore a different certificate. Suppose now that the bottom left PKI (in Figure 9) had double arrows between $Y$ and $C$, as well as between $Y$ and $A$. The following path could then be built:

$$
\text { TA } \quad \mathrm{Z}->\mathrm{BCA}->\mathrm{Y}->\mathrm{A}->\mathrm{C}->\mathrm{Y}->\mathrm{BCA}->\mathrm{W}->\mathrm{BCA}->\mathrm{X}->\mathrm{L}->\mathrm{N}->\mathrm{EE}
$$

A path such as this could become arbitrarily complex and traverse every cross-certified CA in every PKI in a cross-certified environment while still remaining compliant with [X.509]. As a practical matter, the path above is not something an application would typically want or need to build for a variety of reasons: 
- First, certification paths like the example above are generally not intended by the PKI designers and should not be necessary in order to validate any given certificate. If a convoluted path such as the example above is required (there is no corresponding simple path) in order to validate a given certificate, this is most likely indicative of a flaw in the PKI design.

- Second, the longer a path becomes, the greater the potential dilution of trust in the certification path. That is, with each successive link in the infrastructure (i.e., certification by CAs and cross-certification between CAs) some amount of assurance may be considered lost.

- Third, the longer and more complicated a path, the less likely it is to validate because of basic constraints, policies or policy constraints, name constraints, CRL availability, or even revocation.

- Lastly, and certainly not least important from a developer's or user's perspective, is performance. Allowing paths like the one above dramatically increases the number of possible paths for every certificate in a mesh or cross-certified environment. Every path built may require one or more of the following: validation of certificate properties, CPU intensive signature validations, CRL retrievals, increased network load, and local memory caching. Eliminating the superfluous paths can greatly improve performance, especially in the case where no path exists.

There is a special case involving certificates with the same distinguished names but differing encodings required by [RFC3280]. This case should not be considered a repeated certificate. See Section 5.4 for more information.

\subsubsection{Introduction to Path-Building Optimization}

How can these superfluous paths be eliminated? Rather than only disallowing identical certificates from repeating, it is recommended that a developer disallow the same public key and subject name pair from being repeated. For maximum flexibility, the subject name should collectively include any subject alternative names. Using this approach, all of the intended and needed paths should be available, and the excess and diluted paths should be eliminated. For example, using this approach, only one path exists from the TA $Z$ to $\mathrm{EE}$ in the diagram above: TA $\mathrm{Z}->\mathrm{BCA}->\mathrm{X}->\mathrm{L}->\mathrm{N}->\mathrm{EE}$. 
Given the simplifying rule of not repeating pairs of subject names (including subject alternative names) and public keys, and only using certificates found in the cACertificate and forward (issuedToThisCA) element of the crosscertificatePair attributes, Figure 10 depicts the forward path-building decision tree from the EE to all reachable nodes in the graph. This is the ideal graph for a path builder attempting to build a path from TA $Z$ to EE.

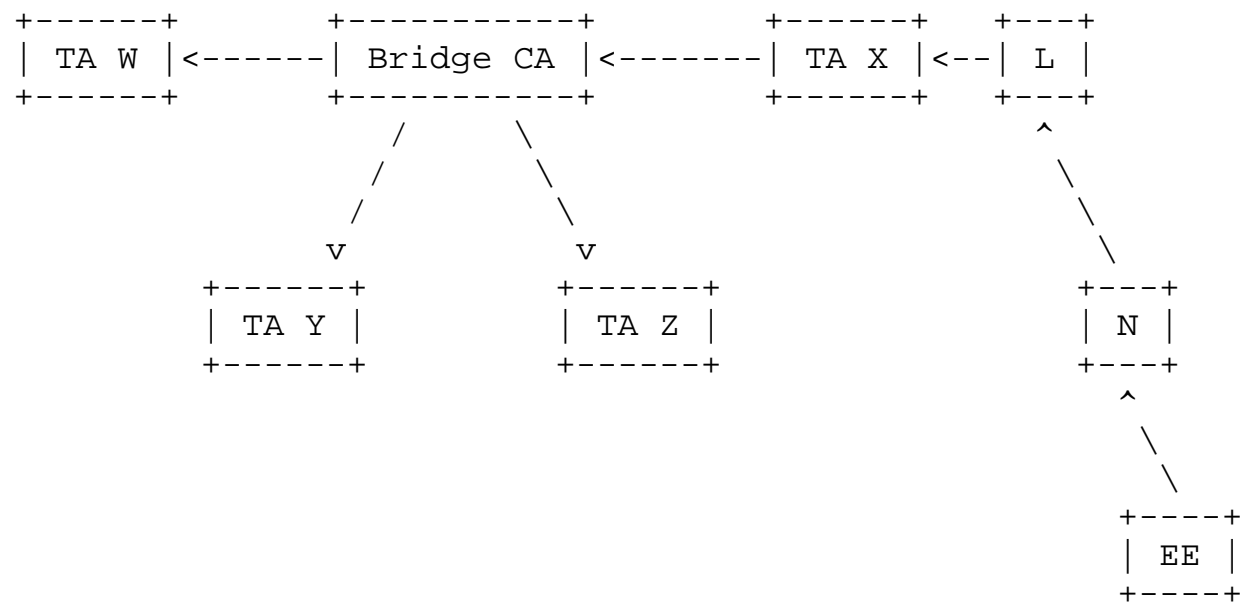

Figure 10 - Forward (From Entity) Decision Tree

It is not possible to build forward direction paths into the infrastructures behind CAs W, Y, and Z, because W, Y, and Z have not been issued certificates by their subordinate CAs. (The subordinate CAs are $F$ and $G, A$ and $C$, and $O$ and $P$, respectively.) If simplicity and speed are desirable, the graph in Figure 10 is a very appealing way to structure the path-building algorithm. Finding a path from the EE to one of the four trust anchors is reasonably simple. Alternately, a developer could choose to build in the opposite direction, using the reverse cross-certificates from any one of the four trust anchors around the BCA. The graph in Figure 11 depicts all possible paths as a tree emanating from TA $Z$. (Note: it is not recommended that implementations attempt to determine all possible paths, this would require retrieval and storage of all PKI data including certificates and CRLs! This example is provided to demonstrate the complexity which might be encountered.) 


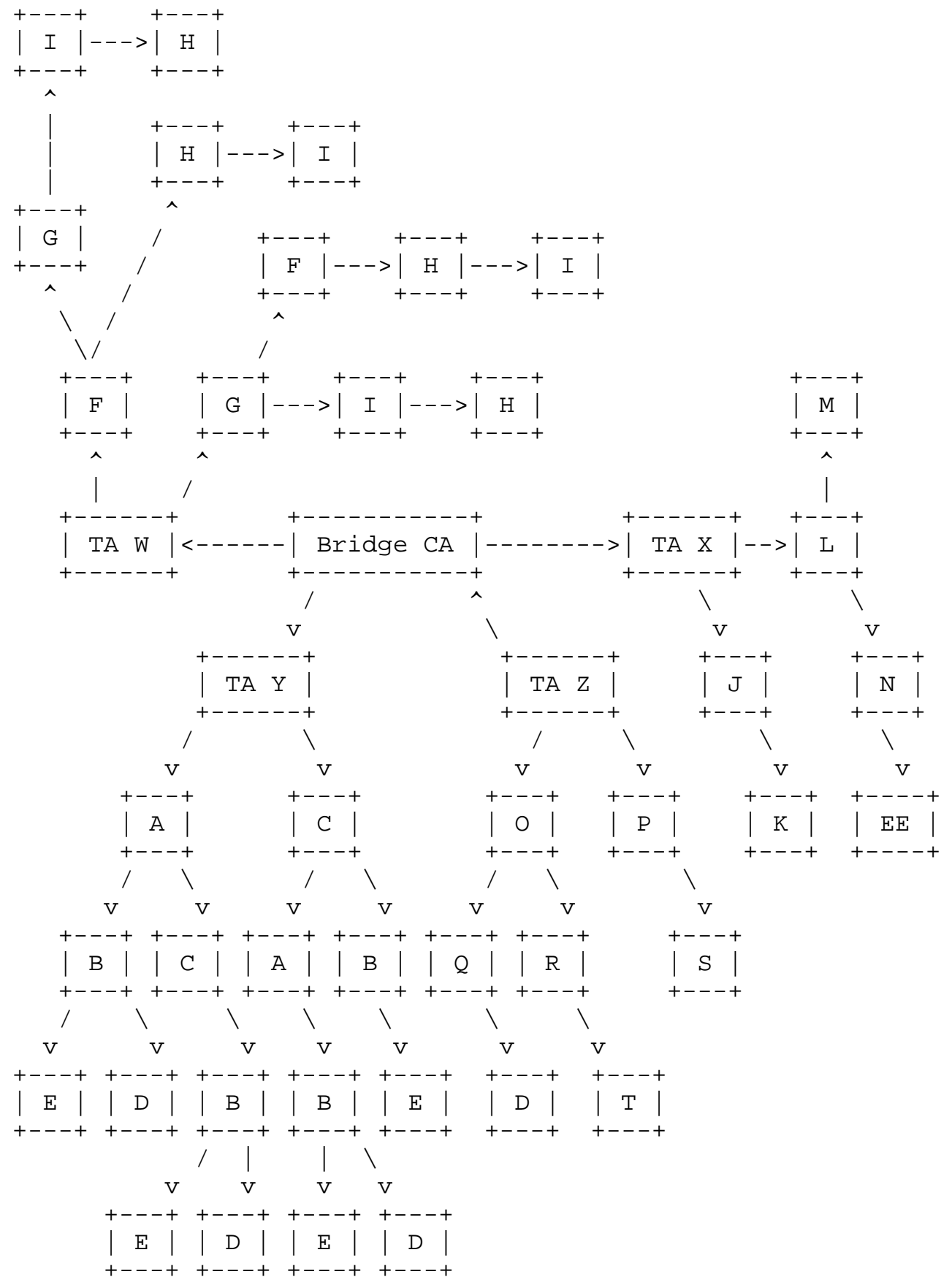

Figure 11 - Reverse (From Anchor) Decision Tree 
Given the relative complexity of this decision tree, it becomes clear that making the right choices while navigating the tree can make a large difference in how quickly a valid path is returned. The pathbuilding software could potentially traverse the entire graph before choosing the shortest path: TA $\mathrm{Z}->\mathrm{BCA}->\mathrm{X}->\mathrm{L}->\mathrm{N}->\mathrm{EE}$. With a decision tree like the one above, the basic depth first traversal approach introduces obvious inefficiencies in the path-building process. To compensate for this, a path-building module needs to decide not only in which direction to traverse the tree, but also which branches of the tree are more likely to yield a valid path.

The path-building algorithm then ideally becomes a tree traversal algorithm with weights or priorities assigned to each branch point to guide the decision making. If properly designed, such an approach would effectively yield the "best path first" more often than not. (The terminology "best path first" is quoted because the definition of the "best" path may differ from PKI to PKI. That is ultimately to be determined by the developer, not by this document.) Finding the "best path first" is an effort to make the implementation efficient, which is one of our criteria as stated in section 2.2.

So how would a developer go about finding the best path first? Given the simplifying idea of addressing path building as a tree traversal, path building could be structured as a depth first search. A simple example of depth first tree traversal path building is depicted in Figure 12, with no preference given to sort order.

Note: The arrows in the lower portion of the figure do not indicate the direction of certificate issuance; they indicate the direction of the tree traversal from the target certificate (EE). 

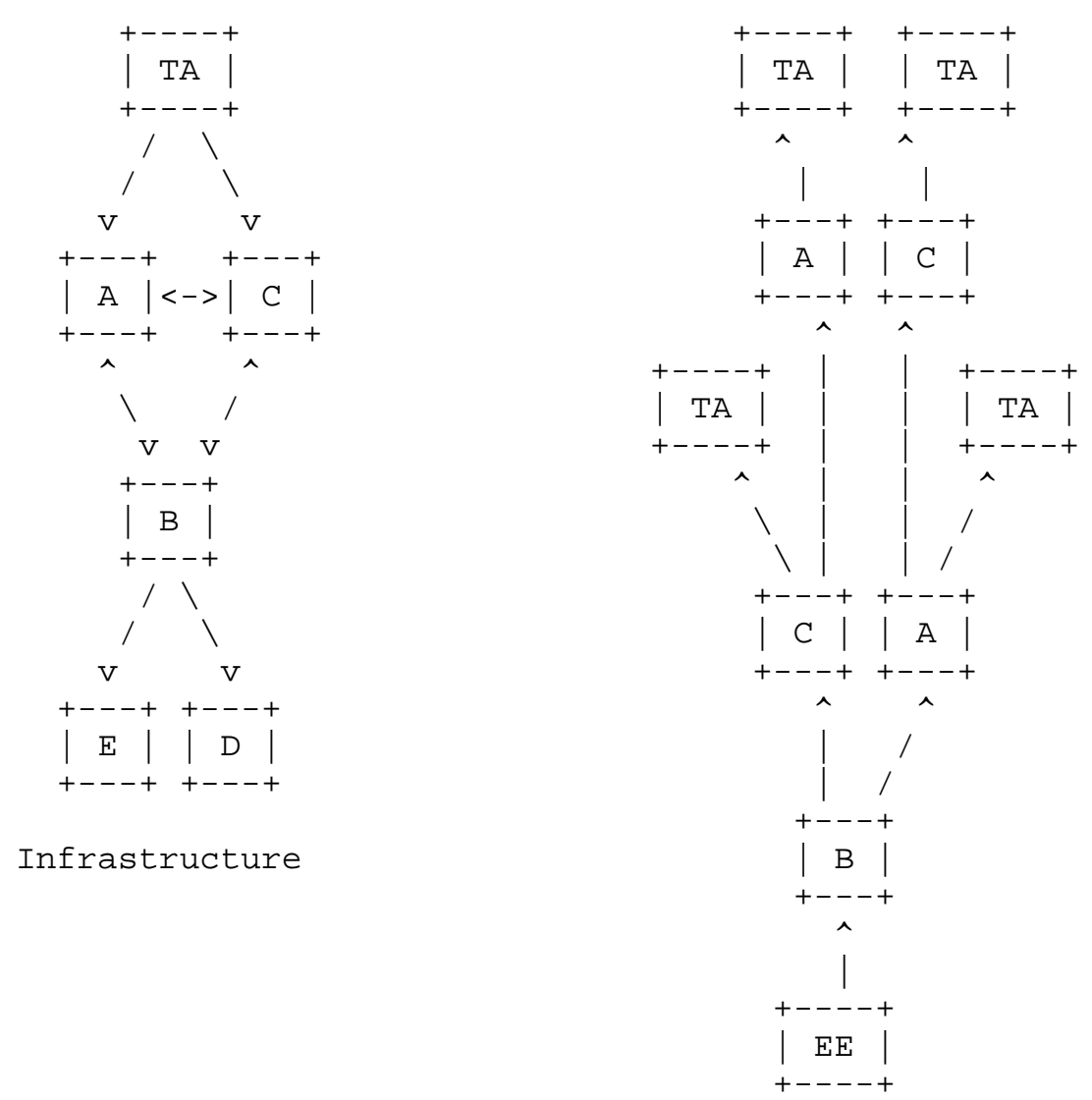

The Same Infrastructure Represented as a Tree 


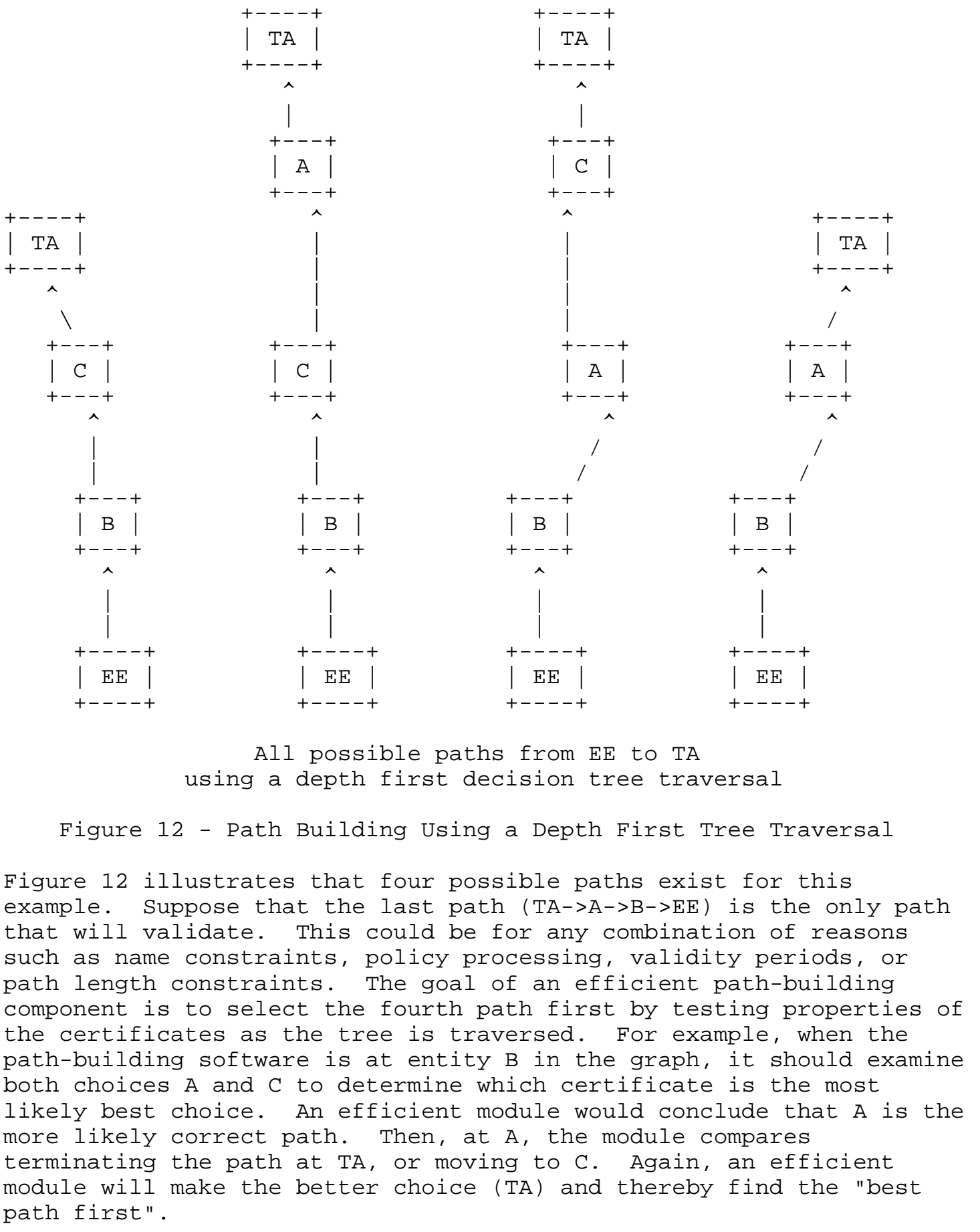


What if the choice between CA certificates is not binary as it was in the previous example? What if the path-building software encounters a branch point with some arbitrary number of $\mathrm{CA}$ certificates thereby creating the same arbitrary number of tree branches? (This would be typical in a mesh style PKI CA, or at a Bridge CA directory entry, as each will have multiple certificates issued to itself from other CAs.) This situation actually does not change the algorithm at all, if it is structured properly. In our example, rather than treating each decision as binary (i.e., choosing A or C), the path-building software should sort all the available possibilities at any given branch point, and then select the best choice from the list. In the event the path could not be built through the first choice, then the second choice should be tried next upon traversing back to that point in the tree. Continue following this pattern until a path is found or all CA nodes in the tree have been traversed. Note that the certificates at any given point in the tree should only be sorted at the time a decision is first made. Specifically, in the example, the sorting of $\mathrm{A}$ and $\mathrm{C}$ is done when the algorithm reached $\mathrm{B}$. There is no memory resident representation of the entire tree. Just like any other recursive depth first search algorithm, the only information the algorithm needs to keep track of is what nodes (entities) in the tree lie behind it on the current path, and for each of those nodes, which arcs (certificates) have already been tried.

\subsection{Building Certification Paths for Revocation Signer Certificates}

Special consideration is given to building a certification path for the Revocation Signer certificate because it may or may not be the same as the Certification Authority certificate. For example, after a CA performs a key rollover, the new CA certificate will be the CRL Signer certificate, whereas the old CA certificate is the Certification Authority certificate for previously issued certificates. In the case of indirect CRLs, the CRL Signer certificate will contain a different name and key than the Certification Authority certificate. In the case of OCSP, the Revocation Signer certificate may represent an OCSP Responder that is not the same entity as the Certification Authority.

When the Revocation Signer certificate and the Certification Authority certificate are identical, no additional consideration is required from a certification path-building standpoint. That is, the certification path built (and validated) for the Certification Authority certificate can also be used as the certification path for the Revocation Signer certificate. In this case, the signature on the revocation data (e.g., CRL or OCSP response) is verified using the same certificate, and no other certification path building is required. An efficient certification path validation algorithm should first try all possible CRLs issued by the Certification 
Authority to determine if any of the CRLs (a) cover the certificate in question, (b) are current, and (c) are signed using the same key used to sign the certificate.

When the Revocation Signer certificate is not identical to the Certification Authority certificate, a certification path must be built (and validated) for the Revocation signer certificate. In general, the certification path-building software may build the path as it would for any other certificate. However, this document also outlines methods in later sections for greatly improving path building efficiency for Revocation Signer certificate case.

\subsection{Suggested Path-Building Software Components}

There is no single way to define an interface to a path-building module. It is not the intent of this document to prescribe a particular method or semantic; rather, it is up to the implementer to decide. There are many ways this could be done. For example, a path-building module could build every conceivable path and return the entire list to the caller. Or, the module could build until it finds just one that validates and then terminate the procedure. Or, it could build paths in an iterative fashion, depending on validation outside of the builder and successive calls to the builder to get more paths until one valid path is found or all possible paths have been found. All of these are possible approaches, and each of these may offer different benefits to a particular environment or application.

Regardless of semantics, a path-building module needs to contain the following components:

1) The logic for building and traversing the certificate graph.

2) Logic for retrieving the necessary certificates (and CRLs and/or other revocation status information if the path is to be validated) from the available source(s).

Assuming a more efficient and agile path-building module is desired, the following is a good starting point and will tie into the remainder of this document. For a path-building module to take full advantage of all the suggested optimizations listed in this document, it will need all of the components listed below.

1) A local certificate and CRL cache.

a. This may be used by all certificate-using components; it does not need to be specific to the path-building software. A local cache could be memory resident, stored in an operating system 
or application certificate store, stored in a database, or even stored in individual files on the hard disk. While the implementation of this cache is beyond the scope of this document, some design considerations are listed below.

2) The logic for building and traversing the certificate graph/tree.

a. This performs sorting functionality for prioritizing certificates (thereby optimizing path building) while traversing the tree.

b. There is no need to build a complete graph prior to commencing path building. Since path building can be implemented as a depth first tree traversal, the path builder only needs to store the current location in the tree along with the points traversed to the current location. All completed branches can be discarded from memory and future branches are discovered as the tree is traversed.

3) Logic for retrieving the necessary certificates from the available certificate source(s):

a. Local cache.

i. Be able to retrieve all certificates for an entity by subject name, as well as individual certificates by issuer and serial number tuple.

ii. Tracking which directory attribute (including issuedToThisCA <forward> and issuedByThisCA <reverse> for split crosscertificatePair attributes) each certificate was found in may be useful. This allows for functionality such as retrieving only forward crosscertificates, etc.

iii. A "freshness" timestamp (cache expiry time) can be used to determine when the directory should be searched again.

b. LDAPV3 directory for certificates and CRLs.

i. Consider supporting multiple directories for general queries.

ii. Consider supporting dynamic LDAP connections for retrieving CRLs using an LDAP URI [RFC3986] in the CRL distribution point certificate extension. 
iii. Support LDAP referrals. This is typically only a matter of activating the appropriate flag in the LDAP API.

c. HTTP support for CRL distribution points and authority information access (AIA) support.

i. Consider HTTPS support, but be aware that this may create an unbounded recursion when the implementation tries to build a certification path for the server's certificate if this in turn requires an additional HTTPS lookup.

4) A certification path cache that stores previously validated relationships between certificates. This cache should include:

a. A configurable expiration date for each entry. This date can be configured based upon factors such as the expiry of the information used to determine the validity of an entry, bandwidth, assurance level, storage space, etc.

b. Support to store previously verified issuer certificate to subject certificate relationships.

i. Since the issuer DN and serial number tuple uniquely identifies a certificate, a pair of these tuples (one for both the issuer and subject) is an effective method of storing this relationship.

c. Support for storing "known bad" paths and certificates. Once a certificate is determined to be invalid, implementations can decide not to retry path development and validation.

2.7. Inputs to the Path-Building Module

[X.509] specifically addresses the list of inputs required for path validation but makes no specific suggestions concerning useful inputs to path building. However, given that the goal of path building is to find certification paths that will validate, it follows that the same inputs used for validation could be used to optimize path building .

\subsubsection{Required Inputs}

Setting aside configuration information such as repository or cache locations, the following are required inputs to the certification path-building process:

1) The Target Certificate: The certificate that is to be validated. This is one endpoint for the path. (It is also possible to 
provide information used to retrieve a certificate for a target, rather than the certificate itself.)

2) Trust List: This is the other endpoint of the path, and can consist of either:

a. Trusted CA certificates

b. Trusted keys and DNs; a certificate is not necessarily required

\subsubsection{Optional Inputs}

In addition to the inputs listed in section 2.7.1, the following optional inputs can also be useful for optimizing path building. However, if the path-building software takes advantage of all of the optimization methods described later in this document, all of the following optional inputs will be required.

1) Time ( $T)$ : The time for which the certificate is to be validated (e.g., if validating a historical signature from one year ago, $\mathrm{T}$ is needed to build a valid path)

a. If not included as an input, the path-building software should always build for $T$ equal to the current system time.

2) Initial-inhibit-policy-mapping indicator

3) Initial-require-explicit-policy indicator

4) Initial-any-policy-inhibit indicator

5) Initial user acceptable policy set

6) Error handlers (call backs or virtual classes)

7) Handlers for custom certificate extensions

8) Is-revocation-provider indicator

a. IMPORTANT: When building a certification path for an OCSP Responder certificate specified as part of the local configuration, this flag should not be set. It is set when building a certification path for a CRL Signer certificate or for an OCSP Responder Signer certificate discovered using the information asserted in an authorityInformationAccess certificate extension. 
9) The complete certification path for the Certification Authority (if Is-revocation-provider is set)

10) Collection of certificates that may be useful in building the path

11) Collection of certificate revocation lists and/or other revocation data

The last two items are a matter of convenience. Alternately, certificates and revocation information could be placed in a local cache accessible to the path-building module prior to attempting to build a path.

3. Optimizing Path Building

This section recommends methods for optimizing path-building processes.

3.1. Optimized Path Building

Path building can be optimized by sorting the certificates at every decision point (at every node in the tree) and then selecting the most promising certificate not yet selected as described in section 2.4.2. This process continues until the path terminates. This is roughly equivalent to the concept of creating a weighted edge tree, where the edges are represented by certificates and nodes represent subject DNs. However, unlike the weighted edge graph concept, a certification path builder need not have the entire graph available in order to function efficiently. In addition, the path builder can be stateless with respect to nodes of the graph not present in the current path, so the working data set can be relatively small.

The concept of statelessness with respect to nodes not in the current path is instrumental to using the sorting optimizations listed in this document. Initially, it may seem that sorting a given group of certificates for a CA once and then preserving that sorted order for later use would be an efficient way to write the path builder. However, maintaining this state can quickly eliminate the efficiency that sorting provides. Consider the following diagram: 


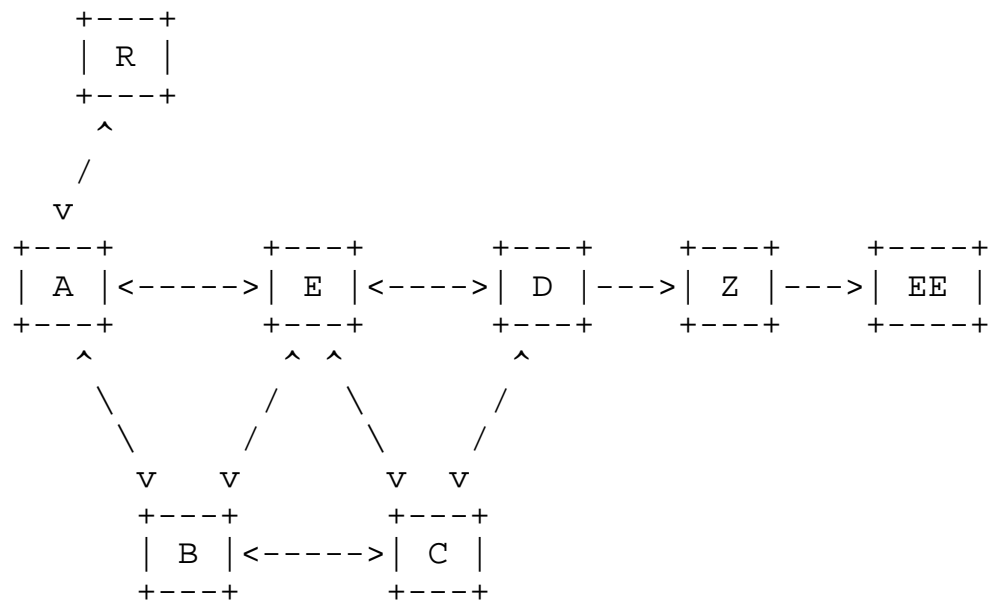

Figure 13 - Example of Path-Building Optimization

In this example, the path builder is building in the forward (from target) direction for a path between $\mathrm{R}$ and $\mathrm{EE}$. The path builder has also opted to allow subject name and key to repeat. (This will allow multiple traversals through any of the cross-certified CAs, creating enough complexity in this small example to illustrate proper state maintenance. Note that a similarly complex example could be designed by using multiple keys for each entity and prohibiting repetition.)

The first step is simple; the builder builds the path Z(D) $\rightarrow$ EE (Z) . Next the builder adds $D$ and faces a decision between two certificates. (Choose between $D(C)$ or $D(E)$ ). The builder now sorts the two choices in order of priority. The sorting is partially based upon what is currently in the path.

Suppose the order the builder selects is [D(E), D(C)]. The current path is now $D(E)->Z(D)->E E(Z)$. Currently the builder has three nodes in the graph (EE, $Z$, and D) and should maintain the state, including sort order of the certificates at $D$, when adding the next node, $E$. When $E$ is added, the builder now has four certificates to sort: $E(A)$, $E(B), E(C)$, and $E(D)$. In this case, the example builder opts for the order $[E(C), E(B), E(A), E(D)]$. The current path is now $E(C)->D(E)->$ $\mathrm{Z}(\mathrm{D})->\mathrm{EE}(\mathrm{Z})$ and the path has four nodes; EE, Z, D, and E.

Upon adding the fifth node, $C$, the builder sorts the certificates $(C(B), C(D)$, and $C(E))$ at $C$, and selects $C(E)$. The path is now $C(E)->E(C)->D(E)->Z(D)->E E(Z)$ and the path has five nodes: EE, Z, D, $E$, and $C$. 
Now the builder finds itself back at node E with four certificates. If the builder were to use the prior sort order from the first encounter with $E$, it would have $[E(C), E(B), E(A), E(D)]$. In the current path's context, this ordering may be inappropriate. To begin with, the certificate $\mathrm{E}(\mathrm{C})$ is already in the path so it certainly does not deserve first place.

The best way to handle this situation is for the path builder to handle this instance of $\mathrm{E}$ as a new (sixth) node in the tree. In other words, there is no state information for this new instance of $E$ - it is treated just as any other new node. The certificates at the new node are sorted based upon the current path content and the first certificate is then selected. For example, the builder may examine $E(B)$ and note that it contains a name constraint prohibiting "C". At this point in the decision tree, $E(B)$ could not be added to the path and produce a valid result since "C" is already in the path. As a result, the certificate $E(B)$ should placed at the bottom of the prioritized list.

Alternatively, $E(B)$ could be eliminated from this new node in the tree. It is very important to see that this certificate is eliminated only at this node and only for the current path. If path building fails through $C$ and traverses back up the tree to the first instance of $E, E(B)$ could still produce a valid path that does not include $C$; specifically $R->A->B->E->D->Z->E E$. Thus the state at any node should not alter the state of previous or subsequent nodes. (Except for prioritizing certificates in the subsequent nodes.)

In this example, the builder should also note that $E(C)$ is already in the path and should make it last or eliminate it from this node since certificates cannot be repeated in a path.

If the builder eliminates both certificates $E(B)$ and $E(C)$ at this node, it is now only left to select between $E(A)$ and $E(D)$. Now the path has six nodes: EE, Z, D, E(1), C, and E(2). E(1) has four certificates, and $E(2)$ has two, which the builder sorts to yield $[E(A), E(D)]$. The current path is now $E(A)->C(E)->E(C)->D(E)->$ $Z(D)->E E(Z)$. A (R) will be found when the seventh node is added to the path and the path terminated because one of the trust anchors has been found.

In the event the first path fails to validate, the path builder will still have the seven nodes and associated state information to work with. On the next iteration, the path builder is able to traverse back up the tree to a working decision point, such as A, and select the next certificate in the sorted list at $A$. In this example, that would be $A(B)$. ( $A(R)$ has already been tested.) This would dead end, and the builder traverse back up to the next decision point, E(2) 
where it would try $D(E)$. This process repeats until the traversal backs all the way up to $\mathrm{EE}$ or a valid path is found. If the tree traversal returns to EE, all possible paths have been exhausted and the builder can conclude no valid path exists.

This approach of sorting certificates in order to optimize path building will yield better results than not optimizing the tree traversal. However, the path-building process can be further streamlined by eliminating certificates, and entire branches of the tree as a result, as paths are built.

\subsection{Sorting vs. Elimination}

Consider a situation when building a path in which three CA certificates are found for a given target certificate and must be prioritized. When the certificates are examined, as in the previous example, one of the three has a name constraint present that will invalidate the path built thus far. When sorting the three certificates, that one would certainly go to the back of the line. However, the path-building software could decide that this condition eliminates the certificate from consideration at this point in the graph, thereby reducing the number of certificate choices by 33\% at this point.

NOTE: It is important to understand that the elimination of a certificate only applies to a single decision point during the tree traversal. The same certificate may appear again at another point in the tree; at that point it may or may not be eliminated. The previous section details an example of this behavior.

Elimination of certificates could potentially eliminate the traversal of a large, time-consuming infrastructure that will never lead to a valid path. The question of whether to sort or eliminate is one that pits the flexibility of the software interface against efficiency.

To be clear, if one eliminates invalid paths as they are built, returning only likely valid paths, the end result will be an efficient path-building module. The drawback to this is that unless the software makes allowances for it, the calling application will not be able to see what went wrong. The user may only see the unrevealing error message: "No certification path found."

On the other hand, the path-building module could opt to not rule out any certification paths. The path-building software could then return any and all paths it can build from the certificate graph. It is then up to the validation engine to determine which are valid and which are invalid. The user or calling application can then have complete details on why each and every path fails to validate. The 
drawback is obviously one of performance, as an application or end user may wait for an extended period of time while cross-certified PKIs are navigated in order to build paths that will never validate.

Neither option is a very desirable approach. One option provides good performance for users, which is beneficial. The other option though allows administrators to diagnose problems with the PKI, directory, or software. Below are some recommendations to reach a middle ground on this issue.

First, developers are strongly encouraged to output detailed log information from the path-building software. The log should explicitly indicate every choice the builder makes and why. It should clearly identify which certificates are found and used at each step in building the path. If care is taken to produce a useful log, PKI administrators and help desk personnel will have ample information to diagnose a problem with the PKI. Ideally, there would be a mechanism for turning this logging on and off, so that it is not running all the time. Additionally, it is recommended that the log contain information so that a developer or tester can recreate the paths tried by the path-building software, to assist with diagnostics and testing.

Secondly, it is desirable to return something useful to the user. The easiest approach is probably to implement a "dual mode" pathbuilding module. In the first mode [mode 1], the software eliminates any and all paths that will not validate, making it very efficient. In the second mode [mode 2], all the sorting methods are still applied, but no paths are eliminated based upon the sorting methods. Having this dual mode allows the module to first fail to find a valid path, but still return one invalid path (assuming one exists) by switching over to the second mode long enough to generate a single path. This provides a middle ground -- the software is very fast, but still returns something that gives the user a more specific error than "no path found".

Third, it may be useful to not rule out any paths, but instead limit the number of paths that may be built given a particular input. Assuming the path-building module is designed to return the "best path first", the paths most likely to validate would be returned before this limit is reached. Once the limit is reached the module can stop building paths, providing a more rapid response to the caller than one which builds all possible paths.

Ultimately, the developer determines how to handle the trade-off between efficiency and provision of information. A developer could choose the middle ground by opting to implement some optimizations as elimination rules and others as not. A developer could validate 
certificate signatures, or even check revocation status while building the path, and then make decisions based upon the outcome of those checks as to whether to eliminate the certificate in question.

This document suggests the following approach:

1) While building paths, eliminate any and all certificates that do not satisfy all path validation requirements with the following exceptions:

a. Do not check revocation status if it requires a directory lookup or network access

b. Do not check digital signatures (see Section 8.1, General Considerations for Building A Certification Path, for additional considerations).

c. Do not check anything that cannot be checked as part of the iterative process of traversing the tree.

d. Create a detailed log, if this feature is enabled.

e. If a path cannot be found, the path builder shifts to "mode 2" and allows the building of a single bad path.

i. Return the path with a failure indicator, as well as error information detailing why the path is bad.

2) If path building succeeds, validate the path in accordance with [X.509] and [RFC3280] with the following recommendations:

a. For a performance boost, do not re-check items already checked by the path builder. (Note: if pre-populated paths are supplied to the path-building system, the entire path has to be fully re-validated.)

b. If the path validation failed, call the path builder again to build another path.

i. Always store the error information and path from the first iteration and return this to the user in the event that no valid path is found. Since the path-building software was designed to return the "best path first", this path should be shown to the user.

As stated above, this document recommends that developers do not validate digital signatures or check revocation status as part of the path-building process. This recommendation is based on two 
assumptions about PKI and its usage. First, signatures in a working PKI are usually good. Since signature validation is costly in terms of processor time, it is better to delay signature checking until a complete path is found and then check the signatures on each certificate in the certification path starting with the trust anchor (see Section 8.1). Second, it is fairly uncommon in typical application environments to encounter a revoked certificate; therefore, most certificates validated will not be revoked. As a result, it is better to delay retrieving CRLs or other revocation status information until a complete path has been found. This reduces the probability of retrieving unneeded revocation status information while building paths.

\subsection{Representing the Decision Tree}

There are a multitude of ways to implement certification path building and as many ways to represent the decision tree in memory.

The method described below is an approach that will work well with the optimization methods listed later in this document. Although this approach is the best the authors of this document have implemented, it is by no means the only way to implement it. Developers should tailor this approach to their own requirements or may find that another approach suits their environment, programming language, or programming style.

\subsubsection{Node Representation for CA Entities}

A "node" in the certification graph is a collection of CA certificates with identical subject DNs. Minimally, for each node, in order to fully implement the optimizations to follow, the pathbuilding module will need to be able to keep track of the following information:

1. Certificates contained in the node

2. Sorted order of the certificates

3. "Current" certificate indicator

4. The current policy set (It may be split into authority and user constrained sets, if desired.)

- It is suggested that encapsulating the policy set in an object with logic for manipulating the set such as performing intersections, mappings, etc., will simplify implementation. 
5. Indicators (requireExplicitpolicy, inhibitpolicyMapping, anyPolicyInhibit) and corresponding skipcert values

6. A method for indicating which certificates are eliminated or removing them from the node.

- If nodes are recreated from the cache on demand, it may be simpler to remove eliminated certificates from the node.

7. A "next" indicator that points to the next node in the current path

8. A "previous" indicator that points to the previous node in the current path

\subsubsection{Using Nodes to Iterate Over All Paths}

In simplest form, a node is created, the certificates are sorted, the next subject DN required is determined from the first certificate, and a new node is attached to the certification path via the next indicator (Number 7 above). This process continues until the path terminates. (Note: end entity certificates may not contain subject DNs as allowed by [RFC3280]. Since end entity certificates by definition do not issue certificates, this has no impact on the process.)

Keeping in mind that the following algorithm is designed to be implemented using recursion, consider the example in Figure 12 and assume that the only path in the diagram is valid for $\mathrm{E}$ is $\mathrm{TA}->\mathrm{A}->$ $B->E$ :

If our path-building module is building a path in the forward direction for $E$, a node is first created for $E$. There are no certificates to sort because only one certificate exists, so all initial values are loaded into the node from E. For example, the policy set is extracted from the certificate and stored in the node.

Next, the issuer DN (B) is read from $E$, and new node is created for $B$ containing both certificates issued to $B--B(A)$ and $B(C)$. The sorting rules are applied to these two certificates and the sorting algorithm returns $B(C) ; B(A)$. This sorted order is stored and the current indicator is set to $B(C)$. Indicators are set and the policy sets are calculated to the extent possible with respect to B(C). The following diagram illustrates the current state with the current certificate indicated with a "*". 


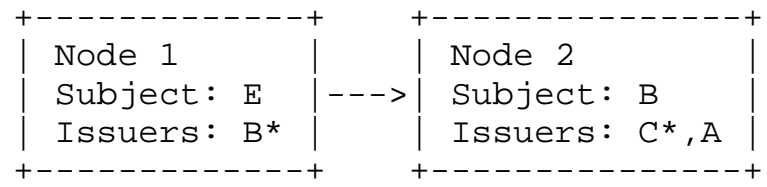

Next, a node is created for $\mathrm{C}$ and all three certificates are added to it. The sorting algorithm happens to return the certificates sorted in the following order: $\mathrm{C}(\mathrm{TA}) ; \mathrm{C}(\mathrm{A})$; C (B)

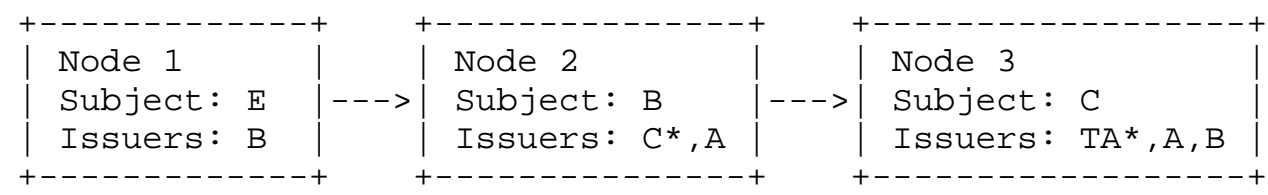

Recognizing that the trust anchor has been found, the path $(\mathrm{TA}->\mathrm{C}->\mathrm{B}->\mathrm{E})$ is validated but fails. (Remember that the only valid path happens to be $\mathrm{TA}->\mathrm{A}->\mathrm{B}->\mathrm{E}$.) The path-building module now moves the current certificate indicator in node 3 to $C(A)$, and adds the node for $A$.

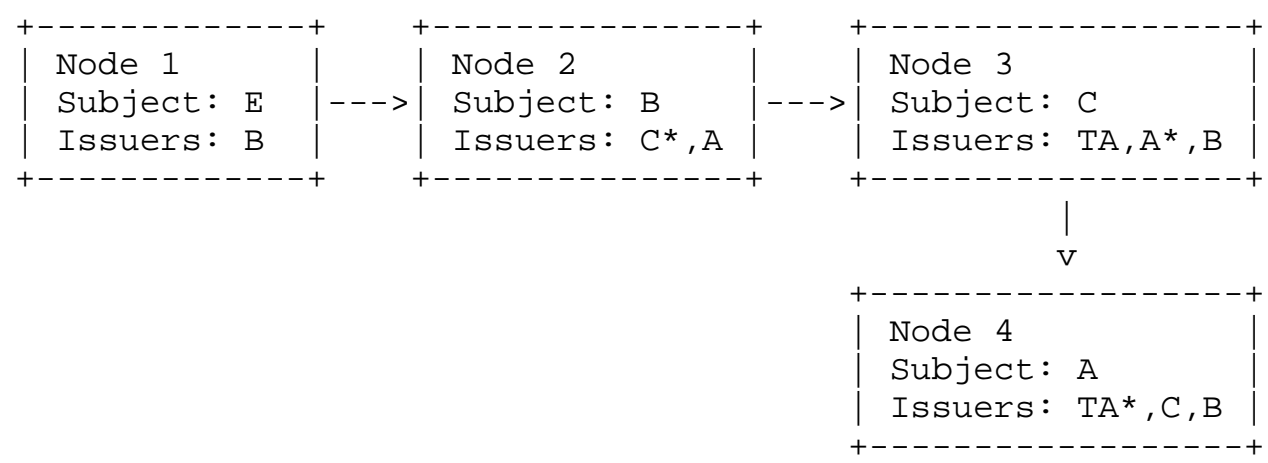

The path $\mathrm{TA}->\mathrm{A}->\mathrm{C}->\mathrm{B}->\mathrm{E}$ is validated and it fails. The path-building module now moves the current indicator in node 4 to $A(C)$ and adds a node for $C$. 


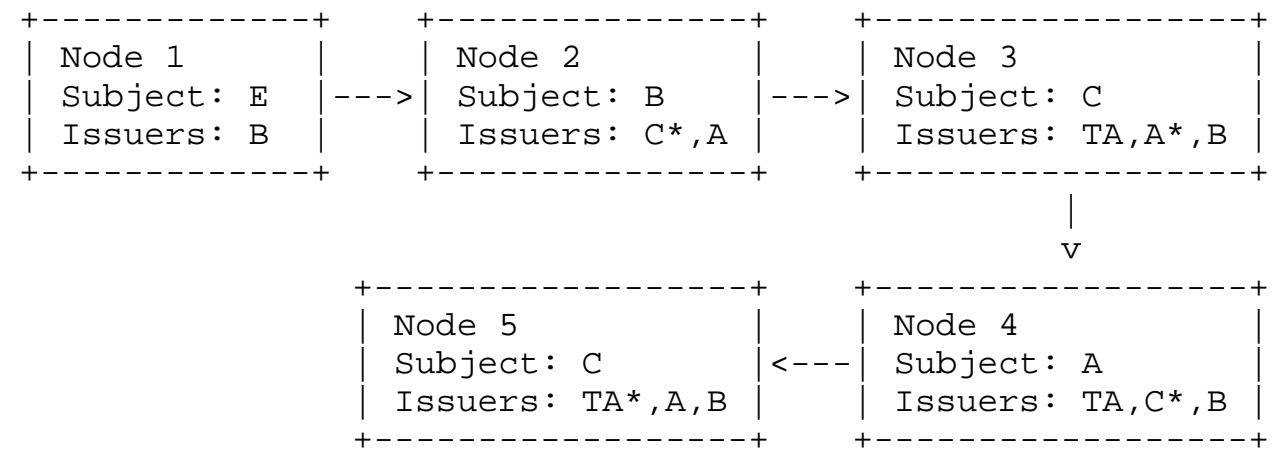

At this juncture, the decision of whether to allow repetition of name and key comes to the forefront. If the certification path-building module will NOT allow repetition of name and key, there are no certificates in node 5 that can be used. ( $C$ and the corresponding public key is already in the path at node 3.) At this point, node 5 is removed from the current path and the current certificate indicator on node 4 is moved to $A(B)$.

If instead, the module is only disallowing repetition of certificates, $C(A)$ is eliminated from node 5 since it is in use in node 3, and path building continues by first validating $\mathrm{TA}->\mathrm{C}->\mathrm{A}->$ $\mathrm{C}->\mathrm{B}->\mathrm{E}$, and then continuing to try to build paths through $\mathrm{C}(\mathrm{B})$. After this also fails to provide a valid path, node 5 is removed from the current path and the current certificate indicator on node 4 is moved to $A(B)$.

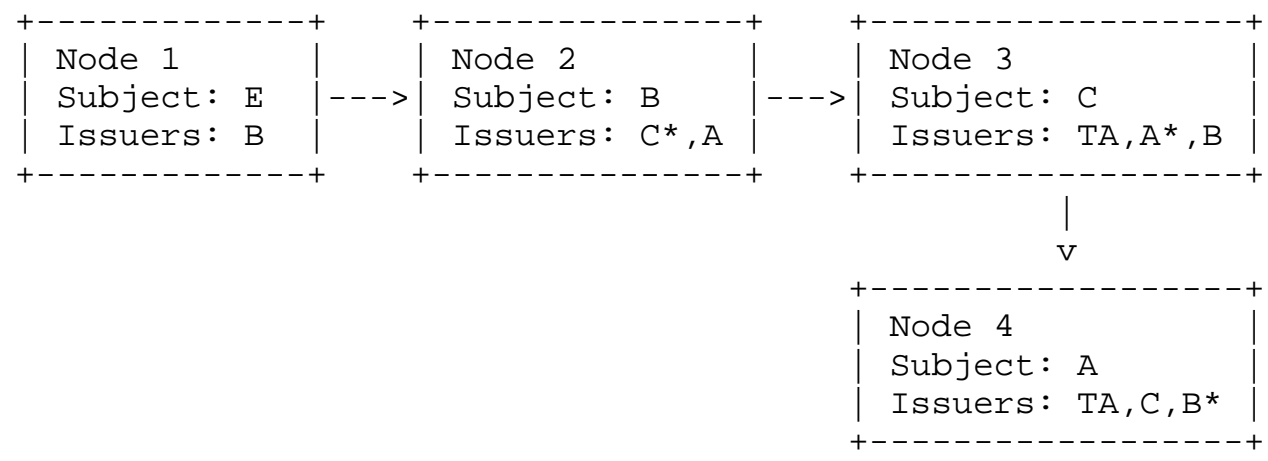

Now a new node 5 is created for B. Just as with the prior node 5, if not repeating name and key, B also offers no certificates that can be used ( $B$ and $B^{\prime}$ s public key is in use in node 2) so the new node 5 is also removed from the path. At this point all certificates in node 4 have now been tried, so node 4 is removed from the path, and the current indicator on node 3 is moved to $C(B)$. 
Also as above, if allowing repetition of name and key, $B(C)$ is removed from the new node 5 (B (C) is already in use in node 3) and paths attempted through the remaining certificate $B(A)$. After this fails, it will lead back to removing node 5 from the path. At this point all certificates in node 4 have now been tried, so node 4 is removed from the path, and the current indicator on node 3 is moved to $C(B)$.

This process continues until all certificates in node 1 (if there happened to be more than one) have been tried, or until a valid path has been found. Once the process ends and in the event no valid path was found, it may be concluded that no path can be found from $\mathrm{E}$ to TA.

\subsection{Implementing Path-Building Optimization}

The following section describes methods that may be used for optimizing the certification path-building process by sorting certificates. Optimization as described earlier seeks to prioritize a list of certificates, effectively prioritizing (weighting) branches of the graph/tree. The optimization methods can be used to assign a cumulative score to each certificate. The process of scoring the certificates amounts to testing each certificate against the optimization methods a developer chooses to implement, and then adding the score for each test to a cumulative score for each certificate. After this is completed for each certificate at a given branch point in the builder's decision tree, the certificates can be sorted so that the highest scoring certificate is selected first, the second highest is selected second, etc.

For example, suppose the path builder has only these two simple sorting methods:

1) If the certificate has a subject key ID, +5 to score.

2) If the certificate has an authority key ID, +10 to score.

And it then examined three certificates:

1) Issued by CA 1; has authority key ID; score is 10.

2) Issued by CA 2; has subject key ID; score is 5.

3) Issued by CA 1; has subject key ID and authority key ID; score is 15 .

The three certificates are sorted in descending order starting with the highest score: 3, 1, and 2. The path-building software should first try building the path through certificate 3. Failing that, it should try certificate 1. Lastly, it should try building a path through certificate 2 . 
The following optimization methods specify tests developers may choose to perform, but does not suggest scores for any of the methods. Rather, developers should evaluate each method with respect to the environment in which the application will operate, and assign weights to each accordingly in the path-building software. Additionally, many of the optimization methods are not binary in nature. Some are tri-valued, and some may be well suited to sliding or exponential scales. Ultimately, the implementer decides the relative merits of each optimization with respect to his or her own software or infrastructure.

Over and above the scores for each method, many methods can be used to eliminate branches during the tree traversal rather than simply scoring and weighting them. All cases where certificates could be eliminated based upon an optimization method are noted with the method descriptions.

Many of the sorting methods described below are based upon what has been perceived by the authors as common in PKIs. Many of the methods are aimed at making path building for the common PKI fast, but there are cases where most any sorting method could lead to inefficient path building. The desired behavior is that although one method may lead the algorithm in the wrong direction for a given situation or configuration, the remaining methods will overcome the errant method(s) and send the path traversal down the correct branch of the tree more often than not. This certainly will not be true for every environment and configuration, and these methods may need to be tweaked for further optimization in the application's target operating environment.

As a final note, the list contained in this document is not intended to be exhaustive. A developer may desire to define additional sorting methods if the operating environment dictates the need.

\subsection{Selected Methods for Sorting Certificates}

The reader should draw no specific conclusions as to the relative merits or scores for each of the following methods based upon the order in which they appear. The relative merit of any sorting criteria is completely dependent on the specifics of the operating environment. For most any method, an example can be created to demonstrate the method is effective and a counter-example could be designed to demonstrate that it is ineffective.

Each sorting method is independent and may (or may not) be used to assign additional scores to each certificate tested. The implementer decides which methods to use and what weights to assign them. As noted previously, this list is also not exhaustive. 
In addition, name chaining (meaning the subject name of the issuer certificate matches the issuer name of the issued certificate) is not addressed as a sorting method since adherence to this is required in order to build the decision tree to which these methods will be applied. Also, unaddressed in the sorting methods is the prevention of repeating certificates. Path builders should handle name chaining and certificate repetition irrespective of the optimization approach.

Each sorting method description specifies whether the method may be used to eliminate certificates, the number of possible numeric values (sorting weights) for the method, components from section 2.6 that are required for implementing the method, forward and reverse methods descriptions, and finally a justification for inclusion of the method.

With regard to elimination of certificates, it is important to understand that certificates are eliminated only at a given decision point for many methods. For example, the path built up to certificate $\mathrm{X}$ may be invalidated due to name constraints by the addition of certificate $Y$. At this decision point only, $Y$ could be eliminated from further consideration. At some future decision point, while building this same path, the addition of $Y$ may not invalidate the path.

For some other sorting methods, certificates could be eliminated from the process entirely. For example, certificates with unsupported signature algorithms could not be included in any path and validated. Although the path builder may certainly be designed to operate in this fashion, it is sufficient to always discard certificates only for a given decision point regardless of cause.

3.5.1. basicConstraints Is Present and CA Equals True

May be used to eliminate certificates: Yes

Number of possible values: Binary

Components required: None

Forward Method: Certificates with basicConstraints present and $C A=T R U E$, or those designated as CA certificates out-of-band have priority. Certificates without basicConstraints, with

basicConstraints and CA=FALSE, or those that are not designated as CA certificates out-of-band may be eliminated or have zero priority.

Reverse Method: Same as forward except with regard to end entity certificates at the terminus of the path.

Justification: According to [RFC3280], basicConstraints is required to be present with $\mathrm{CA}=\mathrm{TRUE}$ in all CA certificates, or must be 
verified via an out-of-band mechanism. A valid path cannot be built if this condition is not met.

3.5.2. Recognized Signature Algorithms

May be used to eliminate certificates: Yes

Number of possible values: Binary

Components required: None

Forward Method: Certificates containing recognized signature and public key algorithms [PKIXALGS] have priority.

Reverse Method: Same as forward.

Justification: If the path-building software is not capable of processing the signatures associated with the certificate, the certification path cannot be validated.

3.5.3. keyUsage Is Correct

May be used to eliminate certificates: Yes Number of possible values: Binary

Components required: None

Forward Method: If keyUsage is present, certificates with keyCertsign set have $100 \%$ priority. If keyUsage is present and keyCertsign is not set, the certificate may be eliminated or have zero priority. All others have zero priority.

Reverse Method: Same as forward except with regard to end entity certificates at the terminus of the path.

Justification: A valid certification path cannot be built through a CA certificate with inappropriate keyUsage. Note that digitalsignature is not required to be set in a CA certificate.

\subsubsection{Time (T) Falls within the Certificate Validity}

May be used to eliminate certificates: Yes

Number of possible values: Binary

Components required: None

Forward Method: Certificates that contain the required time $(T)$ within their validity period have 100\% priority. Otherwise, the certificate is eliminated or has priority zero.

Reverse Method: Same as forward. 
Justification: A valid certification path cannot be built if $\mathrm{T}$ falls outside of the certificate validity period.

NOTE: Special care should be taken to return a meaningful error to the caller, especially in the event the target certificate does not meet this criterion, if this sorting method is used for elimination. (e.g., the certificate is expired or is not yet valid).

\subsubsection{Certificate Was Previously Validated}

May be used to eliminate certificates: No Number of possible values: Binary

Components required: Certification Path Cache

Forward Method: A certificate that is present in the certification path cache has priority.

Reverse Method: Does not apply. (The validity of a certificate vs. unknown validity does not infer anything about the correct direction in the decision tree. In other words, knowing the validity of a CA certificate does not indicate that the target is more likely found through that path than another.)

Justification: Certificates in the path cache have been validated previously. Assuming the initial constraints have not changed, it is highly likely that the path from that certificate to a trust anchor is still valid. (Changes to the initial constraints may cause a certificate previously considered valid to no longer be considered valid.)

Note: It is important that items in the path cache have appropriate life times. For example, it could be inappropriate to cache a relationship beyond the period the related CRL will be trusted by the application. It is also critical to consider certificates and CRLs farther up the path when setting cache lifetimes. For example, if the issuer certificate expires in ten days, but the issued certificate is valid for 20 days, caching the relationship beyond 10 days would be inappropriate.

\subsubsection{Previously Verified Signatures}

May be used to eliminate certificates: Yes

Number of possible values: Binary

Components required: Path Cache

Forward Method: If a previously verified relationship exists in the path cache between the subject certificate and a public key present in one or more issuer certificates, all the certificates containing 
said public key have higher priority. Other certificates may be eliminated or set to zero priority.

Reverse Method: If known bad signature relationships exist between certificates, these relationships can be used to eliminate potential certificates from the decision tree. Nothing can be concluded about the likelihood of finding a given target certificate down one branch versus another using known good signature relationships.

Justification: If the public key in a certificate (A) was previously used to verify a signature on a second certificate (B), any and all certificates containing the same key as (A) may be used to verify the signature on (B). Likewise, any certificates that do not contain the same key as (A) cannot be used to verify the signature on (B). This forward direction method is especially strong for multiply crosscertified CAs after a key rollover has occurred.

\subsubsection{Path Length Constraints}

May be used to eliminate certificates: Yes

Number of possible values: Binary

Components required: None

Forward Method: Certificates with basic constraints present and containing a path length constraint that would invalidate the current path (the current length is known since the software is building from the target certificate) may be eliminated or set to zero priority. Otherwise, the priority is $100 \%$.

Reverse Method: This method may be applied in reverse. To apply it, the builder keeps a current path length constraint variable and then sets zero priority for (or eliminates) certificates that would violate the constraint.

Justification: A valid path cannot be built if the path length constraint has been violated.

\subsubsection{Name Constraints}

May be used to eliminate certificates: Yes Number of possible values: Binary Components required: None

Forward Method: Certificates that contain nameConstraints that would be violated by certificates already in the path to this point are given zero priority or eliminated. 
Reverse Method: Certificates that will allow successful processing of any name constraints present in the path to this point are given higher priority.

Justification: A valid path cannot be built if name constraints are violated.

\subsubsection{Certificate Is Not Revoked}

May be used to eliminate certificates: No Number of possible values: Three Components required: CRL Cache

Forward Method: If a current CRL for a certificate is present in the CRL cache, and the certificate serial number is not on the CRL, the certificate has priority. If the certificate serial number is present on the CRL, it has zero priority. If an (acceptably fresh) OCSP response is available for a certificate, and identifies the certificate as valid, the certificate has priority. If an OCSP response is available for a certificate, and identifies the certificate as invalid, the certificate has zero priority.

Reverse Method: Same as Forward.

Alternately, the certificate may be eliminated if the CRL or OCSP response is verified. That is, fully verify the CRL or OCSP response signature and relationship to the certificate in question in accordance with [RFC3280]. While this is viable, the signature verification required makes it less attractive as an elimination method. It is suggested that this method only be used for sorting and that CRLs and OCSP responses are validated post path building.

Justification: Certificates known to be not revoked can be considered more likely to be valid than certificates for which the revocation status is unknown. This is further justified if CRL or OCSP response validation is performed post path validation - CRLs or OCSP responses are only retrieved when complete paths are found.

NOTE: Special care should be taken to allow meaningful errors to propagate to the caller, especially in cases where the target certificate is revoked. If a path builder eliminates certificates using CRLs or OCSP responses, some status information should be preserved so that a meaningful error may be returned in the event no path is found. 


\subsubsection{Issuer Found in the Path Cache}

May be used to eliminate certificates: No

Number of possible values: Binary

Components required: Certification Path Cache

Forward Method: A certificate whose issuer has an entry (or entries) in the path cache has priority.

Reverse Method: Does not apply.

Justification: Since the path cache only contains entries for certificates that were previously validated back to a trust anchor, it is more likely than not that the same or a new path may be built from that point to the (or one of the) trust anchor(s). For certificates whose issuers are not found in the path cache, nothing can be concluded.

NOTE: This method is not the same as the method named "Certificate Was Previously Validated". It is possible for this sorting method to evaluate to true while the other method could evaluate to zero.

3.5.11. Issuer Found in the Application Protocol

May be used to eliminate certificates: No

Number of possible values: Binary

Components required: Certification Path Cache

Forward Method: If the issuer of a certificate sent by the target through the application protocol (SSL/TLS, S/MIME, etc.), matches the signer of the certificate you are looking at, then that certificate has priority.

Reverse Method: If the subject of a certificate matches the issuer of a certificate sent by the target through the application protocol (SSL/TLS, S/MIME, etc.), then that certificate has priority.

Justification: The application protocol may contain certificates that the sender considers valuable to certification path building, and are more likely to lead to a path to the target certificate.

\subsubsection{Matching Key Identifiers (KIDs)}

May be used to eliminate certificates: No Number of possible values: Three Components required: None

Forward Method: Certificates whose subject key identifier (SKID) 
matches the current certificate's authority key identifier (AKID) have highest priority. Certificates without a SKID have medium priority. Certificates whose SKID does not match the current certificate's AKID (if both are present) have zero priority. If the current certificate expresses the issuer name and serial number in the AKID, certificates that match both these identifiers have highest priority. Certificates that match only the issuer name in the AKID have medium priority.

Reverse Method: Certificates whose AKID matches the current certificate's SKID have highest priority. Certificates without an AKID have medium priority. Certificates whose AKID does not match the current certificate's SKID (if both are present) have zero priority. If the certificate expresses the issuer name and serial number in the AKID, certificates that match both these identifiers in the current certificate have highest priority. Certificates that match only the issuer name in the AKID have medium priority.

Justification: Key Identifier (KID) matching is a very useful mechanism for guiding path building (that is their purpose in the certificate) and should therefore be assigned a heavy weight.

NOTE: Although required to be present by [RFC3280], it is extremely important that KIDs be used only as sorting criteria or as hints during certification path building. KIDs are not required to match during certification path validation and cannot be used to eliminate certificates. This is of critical importance for interoperating across domains and multi-vendor implementations where the KIDs may not be calculated in the same fashion.

\subsubsection{Policy Processing}

May be used to eliminate certificates: Yes Number of possible values: Three Components required: None

Forward Method: Certificates that satisfy Forward Policy Chaining have priority. (See Section 4 entitled "Forward Policy Chaining" for details.) If the caller provided an initial-policy-set and did not set the initial-require-explicit flag, the weight of this sorting method should be increased. If the initial-require-explicit-policy flag was set by the caller or by a certificate, certificates may be eliminated.

Reverse Method: Certificates that contain policies/policy mappings that will allow successful policy processing of the path to this point have priority. If the caller provided an initial-policy-set and did not set the initial-require-explicit flag, the weight of this 
sorting method should be increased. Certificates may be eliminated only if initial-require-explicit was set by the caller or if require-explicit-policy was set by a certificate in the path to this point.

Justification: In a policy-using environment, certificates that successfully propagate policies are more likely part of an intended certification path than those that do not.

When building in the forward direction, it is always possible that a certificate closer to the trust anchor will set the requireexplicit-policy indicator; so giving preference to certification paths that propagate policies may increase the probability of finding a valid path first. If the caller (or a certificate in the current path) has specified or set the initial-require-explicit-policy indicator as true, this sorting method can also be used to eliminate certificates when building in the forward direction.

If building in reverse, it is always possible that a certificate farther along the path will set the require-explicit-policy indicator; so giving preference to those certificates that propagate policies will serve well in that case. In the case where requireexplicit-policy is set by certificates or the caller, certificates can be eliminated with this method.

\subsubsection{Policies Intersect the Sought Policy set}

May be used to eliminate certificates: No

Number of possible values: Additive

Components required: None

Forward Method: Certificates that assert policies found in the initial-acceptable-policy-set have priority. Each additional matching policy could have an additive affect on the total score.

Alternately, this could be binary; it matches 1 or more, or matches none.

Reverse Method: Certificates that assert policies found in the target certificate or map policies to those found in the target certificate have priority. Each additional matching policy could have an additive affect on the total score. Alternately, this could be binary; it matches 1 or more, or matches none.

Justification: In the forward direction, as the path draws near to the trust anchor in a cross-certified environment, the policies asserted in the CA certificates will match those in the caller's domain. Since the initial acceptable policy set is specified in the 
caller's domain, matches may indicate that the path building is drawing nearer to a desired trust anchor. In the reverse direction, finding policies that match those of the target certificate may indicate that the path is drawing near to the target's domain.

\subsubsection{Endpoint Distinguished Name (DN) Matching}

May be used to eliminate certificates: No

Number of possible values: Binary

Components required: None

Forward Method: Certificates whose issuer exactly matches a trust anchor subject DN have priority.

Reverse Method: Certificates whose subject exactly matches the target entity issuer DN have priority.

Justification: In the forward direction, if a certificate's issuer DN matches a trust anchor's DN [X.501], then it may complete the path. In the reverse direction, if the certificate's subject DN matches the issuer DN of the target certificate, it may be the last certificate required to complete the path.

\subsubsection{Relative Distinguished Name (RDN) Matching}

May be used to eliminate certificates: No Number of possible values: Sliding Scale Components required: None

Forward Method: Certificates that match more ordered RDNs between the issuer DN and a trust anchor DN have priority. When all the RDNs match, this yields the highest priority.

Reverse Method: Certificates with subject DNs that match more RDNs with the target's issuer DN have higher priority. When all the RDNs match, this yields the highest priority.

Justification: In PKIs the DNs are frequently constructed in a tree like fashion. Higher numbers of matches may indicate that the trust anchor is to be found in that direction within the tree. Note that in the case where all the RDNs match [X.501], this sorting method appears to mirror the preceding one. However, this sorting method should be capable of producing a 100\% weight even if the issuer DN has more RDNs than the trust anchor. The Issuer DN need only contain all the RDNs (in order) of the trust anchor.

NOTE: In the case where all RDNs match, this sorting method mirrors the functionality of the preceding one. This allows for partial 
matches to be weighted differently from exact matches. Additionally, this method can require a lot of processing if many trust anchors are present.

\subsubsection{Certificates are Retrieved from cACertificate Directory} Attribute

May be used to eliminate certificates: No Number of possible values: Binary Components required: Certificate Cache with flags for the attribute from where the certificate was retrieved and Remote certificate Storage/Retrieval using a directory

Forward Method: Certificates retrieved from the cACertificate directory attribute have priority over certificates retrieved from the crosscertificatePair attribute. (See [RFC2587].)

Reverse Method: Does not apply.

Justification: The cACertificate directory attribute contains certificates issued from local sources and self issued certificates. By using the cACertificate directory attribute before the crosscertificatePair attribute, the path-building algorithm will (depending on the local PKI configuration) tend to demonstrate a preference for the local PKI before venturing to external crosscertified PKIs. Most of today's PKI applications spend most of their time processing information from the local (user's own) PKI, and the local PKI is usually very efficient to traverse due to proximity and network speed.

3.5.18. Consistent Public Key and Signature Algorithms

May be used to eliminate certificates: Yes

Number of possible values: Binary

Components required: None

Forward Method: If the public key in the issuer certificate matches the algorithm used to sign the subject certificate, then it has priority. (Certificates with unmatched public key and signature algorithms may be eliminated.)

Reverse Method: If the public key in the current certificate matches the algorithm used to sign the subject certificate, then it has priority. (Certificates with unmatched public key and signature algorithms may be eliminated.)

Justification: Since the public key and signature algorithms are not consistent, the signature on the subject certificate will not verify 
successfully. For example, if the issuer certificate contains an RSA public key, then it could not have issued a subject certificate signed with the DSA-with-SHA-1 algorithm.

\subsubsection{Similar Issuer and Subject Names}

May be used to eliminate certificates: No

Number of possible values: Sliding Scale

Components required: None

Forward Method: Certificates encountered with a subject DN that matches more RDNs with the issuer DN of the target certificate have priority.

Reverse Method: Same as forward.

Justification: As it is generally more efficient to search the local domain prior to branching to cross-certified domains, using certificates with similar names first tends to make a more efficient path builder. Cross-certificates issued from external domains will generally match fewer RDNs (if any), whereas certificates in the local domain will frequently match multiple RDNs.

\subsubsection{Certificates in the Certification Cache}

May be used to eliminate certificates: No Number of possible values: Three Components required: Local Certificate Cache and Remote Certificate Storage/Retrieval (e.g., LDAP directory as the repository)

Forward Method: A certificate whose issuer certificate is present in the certificate cache and populated with certificates has higher priority. A certificate whose issuer's entry is fully populated with current data (all certificate attributes have been searched within the timeout period) has higher priority.

Reverse Method: If the subject of a certificate is present in the certificate cache and populated with certificates, then it has higher priority. If the entry is fully populated with current data (all certificate attributes have been searched within the timeout period) then it has higher priority.

Justification: The presence of required directory values populated in the cache increases the likelihood that all the required certificates and CRLs needed to complete the path from this certificate to the trust anchor (or target if building in reverse) are present in the cache from a prior path being developed, thereby 
eliminating the need for directory access to complete the path. In the event no path can be found, the performance cost is low since the certificates were likely not retrieved from the network.

\subsubsection{Current CRL Found in Local Cache}

May be used to eliminate certificates: No

Number of possible values: Binary

Components Required: CRL Cache

Forward Method: Certificates have priority if the issuer's CRL entry exists and is populated with current data in the CRL cache.

Reverse Method: Certificates have priority if the subject's CRL entry exists and is populated with current data in the CRL cache.

Justification: If revocation is checked only after a complete path has been found, this indicates that a complete path has been found through this entity at some past point, so a path still likely exists. This also helps reduce remote retrievals until necessary.

3.6. Certificate Sorting Methods for Revocation Signer Certification Paths

Unless using a locally-configured OCSP responder or some other locally-configured trusted revocation status service, certificate revocation information is expected to be provided by the PKI that issued the certificate. It follows that when building a certification path for a Revocation Signer certificate, it is desirable to confine the building algorithm to the PKI that issued the certificate. The following sorting methods seek to order possible paths so that the intended Revocation Signer certification path is found first.

These sorting methods are not intended to be used in lieu of the ones described in the previous section; they are most effective when used in conjunction with those in section 3.5. Some sorting criteria below have identical names as those in the preceding section. This indicates that the sorting criteria described in the preceding section are modified slightly when building the Revocation signer certification path.

\subsubsection{Identical Trust Anchors}

May be used to eliminate certificates: No Number of possible values: Binary Components required: Is-revocation-signer indicator and the Certification Authority's trust anchor 
Forward Method: Not applicable.

Reverse Method: Path building should begin from the same trust anchor used to validate the Certification Authority before trying any other trust anchors. If any trust anchors exist with a different public key but an identical subject DN to that of the Certification Authority's trust anchor, they should be tried prior to those with mismatched names.

Justification: The revocation information for a given certificate should be produced by the PKI that issues the certificate. Therefore, building a path from a different trust anchor than the Certification Authority's is not desirable.

3.6.2. Endpoint Distinguished Name (DN) Matching

May be used to eliminate certificates: No Number of possible values: Binary Components required: Is-revocation-signer indicator and the Certification Authority's trust anchor

Forward Method: Operates identically to the sorting method described in 3.5.15, except that instead of performing the matching against all trust anchors, the DN matching is performed only against the trust anchor DN used to validate the CA certificate.

Reverse Method: No change for Revocation Signer's certification path.

Justification: The revocation information for a given certificate should be produced by the PKI that issues the certificate.

Therefore, building a path to a different trust anchor than the $\mathrm{CA}^{\prime} \mathrm{S}$ is not desirable. This sorting method helps to guide forward direction path building toward the trust anchor used to validate the CA certificate.

\subsubsection{Relative Distinguished Name (RDN) Matching}

May be used to eliminate certificates: No Number of possible values: Sliding scale Components required: Is-revocation-signer indicator and the Certification Authority's trust anchor

Forward Method: Operates identically to the sorting method described in 3.5.16 except that instead of performing the RDN matching against all trust anchors, the matching is performed only against the trust anchor DN used to validate the CA certificate. 
Reverse Method: No change for Revocation Signer's certification path.

Justification: The revocation information for a given certificate should be produced by the PKI that issues the certificate. Therefore, building a path to a different trust anchor than the $\mathrm{CA}^{\prime} \mathrm{s}$ is not desirable. This sorting method helps to guide forward direction path building toward the trust anchor used to validate the CA certificate.

\subsubsection{Identical Intermediate Names}

May be used to eliminate certificates: No Number of possible values: Binary Components required: Is-revocation-signer indicator and the Certification Authority's complete certification path

Forward Method: If the issuer DN in the certificate matches the issuer DN of a certificate in the Certification Authority's path, it has higher priority.

Reverse Method: If the subject DN in the certificate matches the subject DN of a certificate in the Certification Authority's path, it has higher priority.

Justification: Following the same path as the Certificate should deter the path-building algorithm from wandering in an inappropriate direction. Note that this sorting method is indifferent to whether the certificate is self-issued. This is beneficial in this situation because it would be undesirable to lower the priority of a re-key certificate.

\section{Forward Policy Chaining}

It is tempting to jump to the conclusion that certificate policies offer little assistance to path building when building from the target certificate. It's easy to understand the "validate as you go" approach from the trust anchor, and much less obvious that any value can be derived in the other direction. However, since policy validation consists of the intersection of the issuer policy set with the subject policy set and the mapping of policies from the issuer set to the subject set, policy validation can be done while building a path in the forward direction as well as the reverse. It is simply a matter of reversing the procedure. That is not to say this is as ideal as policy validation when building from the trust anchor, but it does offer a method that can be used to mostly eliminate what has long been considered a weakness inherent to building in the forward (from the target certificate) direction. 


\subsection{Simple Intersection}

The most basic form of policy processing is the intersection of the policy sets from the first CA certificate through the target certificate. Fortunately, the intersection of policy sets will always yield the same final set regardless of the order of intersection. This allows processing of policy set intersections in either direction. For example, if the trust anchor issues a CA certificate (A) with policies $\{X, Y, Z\}$, and that CA issues another CA certificate (B) with policies $\{X, Y\}$, and CA B then issues a third CA certificate (C) with policy set $\{Y, G\}$, one normally calculates the policy set from the trust anchor as follows:

1) Intersect $A\{X, Y, Z\}$ with $B\{X, Y\}$ to yield the set $\{X, Y\}$

2) Intersect that result, $\{X, Y\}$ with $C\{Y, G\}$ to yield the final set $\{\mathrm{Y}\}$

Now it has been shown that certificate C is good for policy $Y$. The other direction is exactly the same procedure, only in reverse:

1) Intersect $\mathrm{C}\{\mathrm{Y}, \mathrm{G}\}$ with $\mathrm{B}\{\mathrm{X}, \mathrm{Y}\}$ to yield the set $\{\mathrm{Y}\}$

2) Intersect that result, $\{Y\}$ with $A\{X, Y, Z\}$ to yield the final set $\{Y\}$

Just like in the reverse direction, it has been shown that certificate $C$ is good for policy $Y$, but this time in the forward direction.

When building in the forward direction, policy processing is handled much like it is in reverse -- the software lends preference to certificates that propagate policies. Neither approach guarantees that a path with valid policies will be found, but rather both approaches help guide the path in the direction it should go in order for the policies to propagate.

If the caller has supplied an initial-acceptable-policy set, there is less value in using it when building in the forward direction unless the caller also set inhibit-policy-mapping. In that case, the path builder can further constrain the path building to propagating policies that exist in the initial-acceptable-policy-set. However, even if the inhibit-policy-mapping is not set, the initial-policy-set can still be used to guide the path building toward the desired trust anchor. 


\subsection{Policy Mapping}

When a CA issues a certificate into another domain, an environment with disparate policy identifiers to its own, the CA may make use of policy mappings to map equivalence from the local domain's policy to the non-local domain's policy. If in the prior example, A had included a policy mapping that mapped $X$ to $G$ in the certificate it issued to B, C would be good for $\mathrm{X}$ and $\mathrm{Y}$ :

1) Intersect $A\{X, Y, Z\}$ with $B\{X, Y\}$ to yield the set $\{X, Y\}$

2) Process Policy Mappings in $B^{\prime}$ s certificate ( $X$ maps to G) to yield $\{G, Y\} \quad$ (same as $\{Y, G\}$ )

3) Intersect that result, $\{G, Y\}$ with $C\{Y, G\}$ to yield the final set $\{G, Y\}$

Since policies are always expressed in the relying party's domain, the certificate $C$ is said to be $\operatorname{good}$ for $\{X, Y\}$, not $\{Y, G\}$. This is because "G" doesn't mean anything in the context of the trust anchor that issued A without the policy mapping.

When building in the forward direction, policies can be "unmapped" by reversing the mapping procedure. This procedure is limited by one important aspect: if policy mapping has occurred in the forward direction, there is no mechanism by which it can be known in advance whether or not a future addition to the current path will invalidate the policy chain (assuming one exists) by setting inhibit-policymapping. Fortunately, it is uncommon practice to set this flag. The following is the procedure for processing policy mapping in the forward direction:

1) Begin with $C^{\prime}$ s policy set $\{Y, G\}$

2) Apply the policy mapping in $B^{\prime} S$ certificate ( $X$ maps to $G$ ) in reverse to yield $\{Y, X\}$ (same as $\{X, Y\}$ )

3) Intersect the result $\{X, Y\}$ with $B\{X, Y\}$ to yield the set $\{X, Y\}$

4) Intersect that result, $\{X, Y\}$, with $A\{X, Y, Z\}$ to yield the final set $\{\mathrm{X}, \mathrm{Y}\}$

Just like in the reverse direction, it is determined in the forward direction that certificate $\mathrm{C}$ is good for policies $\{\mathrm{X}, \mathrm{Y}\}$. If during this procedure, an inhibit-policy-mapping flag was encountered, what should be done? This is reasonably easy to keep track of as well. The software simply maintains a flag on any policies that were propagated as a result of a mapping; just a simple Boolean kept with 
the policies in the set. Imagine now that the certificate issued to A has the inhibit-policy-mapping constraint expressed with a skip certificates value of zero.

1) Begin with $C^{\prime}$ s policy set $\{Y, G\}$

2) Apply the policy mapping in $B^{\prime}$ s certificate and mark $X$ as resulting from a mapping. ( $X$ maps to $G$ ) in reverse to yield $\{Y, X m\}$ (same as $\{X m, Y\}$ )

3) Intersect the result $\{X m, Y\}$ with $B\{X, Y\}$ to yield the set $\{X m, Y\}$

4) A's certificate expresses the inhibit policy mapping constraint, so eliminate any policies in the current set that were propagated due to mapping (which is $\mathrm{Xm}$ ) to yield $\{\mathrm{Y}\}$

5) Intersect that result, $\{Y\}$ with $A\{X, Y, Z\}$ to yield the final set $\{\mathrm{Y}\}$

If in our example, the policy set had gone to empty at any point (and require-explicit-policy was set), the path building would back up and try to traverse another branch of the tree. This is analogous to the path-building functionality utilized in the reverse direction when the policy set goes to empty.

\subsection{Assigning Scores for Forward Policy Chaining}

Assuming the path-building module is maintaining the current forward policy set, weights may be assigned using the following procedure:

1) For each CA certificate being scored:

a. Copy the current forward policy set.

b. Process policy mappings in the CA certificate in order to "un-map" policies, if any.

c. Intersect the resulting set with CA certificate's policies.

The larger the policy set yielded, the larger the score for that CA certificate.

2) If an initial acceptable set was supplied, intersect this set with the resulting set for each $\mathrm{CA}$ certificate from (1).

The larger the resultant set, the higher the score is for this certificate. 
Other scoring schemes may work better if the operating environment dictates.

\section{Avoiding Path-Building Errors}

This section defines some errors that may occur during the pathbuilding process, as well as ways to avoid these errors when developing path-building functions.

\subsection{Dead Ends}

When building certification paths in a non-hierarchical PKI structure, a simple path-building algorithm could fail prematurely without finding an existing path due to a "dead end". Consider the example in Figure 14 .

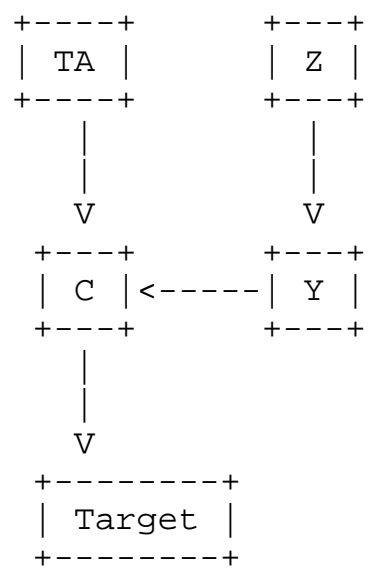

Figure 14 - Dead End Example

Note that in the example, $\mathrm{C}$ has two certificates: one issued by $\mathrm{Y}$, and the other issued by the Trust Anchor. Suppose that a simple "find issuer" algorithm is used, and the order in which the path builder found the certificates was Target(C), C(Y), Y(Z), Z(Z). In this case, $Z$ has no certificates issued by any other entities, and so the simplistic path-building process stops. Since $Z$ is not the relying party's trust anchor, the certification path is not complete, and will not validate. This example shows that in anything but the simplest PKI structure, additional path-building logic will need to handle the cases in which entities are issued multiple certificates from different issuers. The path-building algorithm will also need to have the ability to traverse back up the decision tree and try another path in order to be robust. 


\subsection{Loop Detection}

In a non-hierarchical PKI structure, a path-building algorithm may become caught in a loop without finding an existing path. Consider the example below:

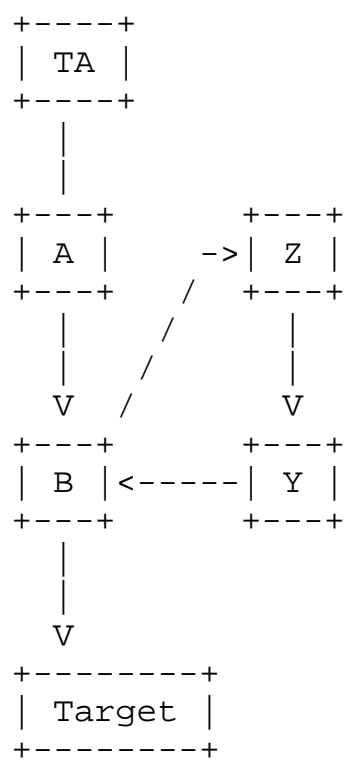

Figure 15 - Loop Example

Let us suppose that in this example the simplest "find issuer" algorithm is used, and the order in which certificates are retrieved is Target $(B), B(Y), Y(Z), Z(B), B(Y), Y(Z), Z(B), B(Y), \ldots A$ loop has formed that will cause the correct path (Target, $B, A$ ) to never be found. The certificate processing system will need to recognize loops created by duplicate certificates (which are prohibited in a path by [X.509]) before they form to allow the certification pathbuilding process to continue and find valid paths. The authors of this document recommend that the loop detection not only detect the repetition of a certificate in the path, but also detect the presence of the same subject name / subject alternative name/ subject public key combination occurring twice in the path. A name/key pair should only need to appear once in the path. (See Section 2.4.2 for more information on the reasoning behind this recommendation.) 
5.3. Use of Key Identifiers

Inconsistent and/or incompatible approaches to computing the subject key identifier and authority key identifier in public key certificates can cause failures in certification path-building algorithms that use those fields to identify certificates, even though otherwise valid certification paths may exist. Path-building implementations should use existing key identifiers and not attempt to re-compute subject key identifiers. It is extremely important that Key Identifiers be used only as sorting criteria or hints. KIDs are not required to match during certification path validation and cannot be used to eliminate certificates. This is of critical importance for interoperating across domains and multi-vendor implementations where the KIDs may not be calculated in the same fashion.

Path-building and processing implementations should not rely on the form of authority key identifier that uses the authority DN and serial number as a restrictive matching rule, because crosscertification can lead to this value not being matched by the crosscertificates.

\subsection{Distinguished Name Encoding}

Certification path-building software should not rely on DNs being encoded as Printablestring. Although frequently encoded as Printablestring, DNs may also appear as other types, including BMPString or UTF8String. As a result, software systems that are unable to process BMPString and UTF8String encoded DNs may be unable to build and validate some certification paths.

Furthermore, [RFC3280] compliant certificates are required to encode DNs as UTF8String as of January 1, 2004. Certification path-building software should be prepared to handle "name rollover" certificates as described in [RFC3280]. Note that the inclusion of a "name rollover" certificate in a certification path does not constitute repetition of a DN and key. Implementations that include the "name rollover" certificate in the path should ensure that the DNs with differing encoding are regarded as dissimilar. (Implementations may instead handle matching DNs of different encodings and will therefore not need to include "name rollover" certificates in the path.) 


\section{Retrieval Methods}

Building a certification path requires the availability of the certificates and CRLs that make up the path. There are many different methods for obtaining these certificates and CRLs. This section lists a few of the common ways to perform this retrieval, as well as some suggested approaches for improving performance. This section is not intended to provide a complete reference for certificate and CRL retrieval methods or optimizations that would be useful in certification path building.

\subsection{Directories Using LDAP}

Most applications utilize the Lightweight Directory Access Protocol (LDAP) when retrieving data from directories following the X.500 model. Applications may encounter directories which support either LDAP v2 [RFC1777] or LDAP v3 [RFC3377].

The LDAP V3 specification defines one attribute retrieval option, the "binary" option. When specified in an LDAP retrieval request, this option was intended to force the directory to ignore any string-based representations of BER-encoded directory information, and send the requested attribute(s) in BER format. Since all PKI objects of concern are BER-encoded objects, the "binary" option should be used. However, not all directories support the "binary" option. Therefore, applications should be capable of requesting attributes with and without the "binary" option. For example, if an application wishes to retrieve the userCertificate attribute, the application should request "userCertificate;binary". If the desired information is not returned, robust implementations may opt to request "userCertificate" as well.

The following attributes should be considered by PKI application developers when performing certificate retrieval from LDAP sources:

userCertificate: contains certificates issued by one or more certification authorities with a subject DN that matches that of the directory entry. This is a multi-valued attribute and all values should be received and considered during path building. Although typically it is expected that only end entity certificates will be stored in this attribute, (e.g., this is the attribute an application would request to find a person's encryption certificate) implementers may opt to search this attribute when looking in CA entries to make their path builder more robust. If it is empty, the overhead added by including this attribute when already requesting one or both of the two below is marginal. 
cACertificate: contains self-issued certificates (if any) and any certificates issued to this certification authority by other certification authorities in the same realm. (Realm is dependent upon local policy.) This is a multi-valued attribute and all values should be received and considered during path building.

crosscertificatePair: in conformant implementations, the crosscertificatePair is used to contain all, except self-issued certificates issued to this certification authority, as well as certificates issued by this certification authority to other certification authorities. Each attribute value is a structure containing two elements. The issuedToThisCA element contains certificates issued to this certification authority by other certification authorities. The issuedByThisCA element contains certificates issued by this certification authority to other certification authorities. Both elements of the crossCertificatePair are labeled optional in the ASN.1 definition. If both elements are present in a single value, the issuer name in one certificate is required to match the subject name in the other and vice versa, and the subject public key in one certificate shall be capable of verifying the digital signature on the other certificate and vice versa. As this technology has evolved, different standards have had differing requirements on where information could be found. For example, the LDAP v2 schema [RFC2587] states that the issuedToThisCA (once called 'forward') element of the crosscertificatePair attribute is mandatory and the issuedByThisCA (once called 'reverse') element is optional. In contrast, Section 11.2.3 of [X.509] requires the issuedByThisCA element to be present if the CA issues a certificate to another CA if the subject is not a subordinate $\mathrm{CA}$ in a hierarchy. Conformant directories behave as required by [X.509], but robust pathbuilding implementations may want to retrieve all certificates from the cACertificate and crosscertificatePair attributes to ensure all possible certification authority certificates are obtained.

certificateRevocationList: the certificateRevocationList attribute contains a certificate revocation list (CRL). A CRL is defined in [RFC3280] as a time stamped list identifying revoked certificates, which is signed by a CA or CRL issuer and made freely available in a public repository. Each revoked certificate is identified in a CRL by its certificate serial number. There may be one or more CRLs in this attribute, and the values should be processed in accordance with [RFC3280]. 
authorityRevocationList: the authorityRevocationList attribute also contains CRLs. These CRLs contain revocation information regarding certificates issued to other CAs. There may be one or more CRLs in this attribute, and the values should be processed in accordance with [RFC3280].

Certification path processing systems that plan to interoperate with varying PKI structures and directory designs should at a minimum be able to retrieve and process the userCertificate, cAcertificate, crosscertificatePair, certificateRevocationList, and

authorityRevocationList attributes from directory entries.

\subsection{Certificate Store Access via HTTP}

Another possible method of certificate retrieval is using HTTP as an interface mechanism for retrieving certificates and CRLs from PKI repositories. A current PKIX document [CERTSTORE] provides a protocol for a general-purpose interface capability for retrieving certificates and CRLs from PKI repositories. Since the [CERTSTORE] document is a work in progress as of the writing of this document, no details are given here on how to utilize this mechanism for certificate and CRL retrieval. Instead, refer to the [CERTSTORE] document or its current version. Certification path processing systems may wish to implement support for this interface capability, especially if they will be used in environments that will provide HTTP-based access to certificates and CRLs.

\subsection{Authority Information Access}

The authority information access (AIA) extension, defined within [RFC3280], indicates how to access CA information and services for the issuer of the certificate in which the extension appears. If a certificate with an AIA extension contains an accessMethod defined with the id-ad-caIssuers OID, the AIA may be used to retrieve one or more certificates for the CA that issued the certificate containing the AIA extension. The AIA will provide a uniform resource identifier (URI) [RFC3986] when certificates can be retrieved via LDAP, HTTP, or FTP. The AIA will provide a directoryName when certificates can be retrieved via directory access protocol (DAP). The AIA will provide an rfc822Name when certificates can be retrieved via electronic mail. Additionally, the AIA may specify the location of an OCSP [RFC2560] responder that is able to provide revocation information for the certificate.

If present, AIA may provide forward path-building implementations with a direct link to a certificate for the issuer of a given certificate. Therefore, implementations may wish to provide support for decoding the AIA extension and processing the LDAP, HTTP, FTP, 
DAP, or e-mail locators. Support for AIA is optional; [RFC3280] compliant implementations are not required to populate the AIA extension. However, implementers of path-building and validation modules are strongly encouraged to support AIA, especially the HTTP transport; this will provide for usability and interoperability with many existing PKIs.

\subsection{Subject Information Access}

The subject information access (SIA) extension, defined within [RFC3280], indicates how to access information and services for the subject of the certificate in which the extension appears. If a certificate with an SIA extension contains an accessMethod defined with the id-ad-caRepository OID, the SIA may be used to locate one or more certificates (and possibly CRLS) for entities issued certificates by the subject. The SIA will provide a uniform resource identifier (URI) [RFC3986] when data can be retrieved via LDAP, HTTP, or FTP. The SIA will provide a directoryName when data can be retrieved via directory access protocol (DAP). The SIA will provide an rfc822Name when data can be retrieved via electronic mail.

If present, the SIA extension may provide reverse path-building implementations with the certificates required to continue building the path. Therefore, implementations may wish to provide support for decoding the SIA extension and processing the LDAP, HTTP, FTP, DAP, or e-mail locators. Support for SIA is optional; [RFC3280] compliant implementations are not required to populate the SIA extension. However, implementers of path-building and validation modules are strongly encouraged to support SIA, especially the HTTP transport; this will provide for usability and interoperability with many existing PKIs.

\subsection{CRL Distribution Points}

The CRL distribution points (CRLDP) extension, defined within [RFC3280], indicates how to access CRL information. If a CRLDP extension appears within a certificate, the CRL(s) to which the CRLDP refer are generally the CRLs that would contain revocation information for the certificate. The CRLDP extension may point to multiple distribution points from which the CRL information may be obtained; the certificate processing system should process the CRLDP extension in accordance with [RFC3280]. The most common distribution points contain URIs from which the appropriate CRL may be downloaded, and directory names, which can be queried in a directory to retrieve the CRL attributes from the corresponding entry. 
If present, CRLDP can provide certificate processing implementations with a link to CRL information for a given certificate. Therefore, implementations may wish to provide support for decoding the CRLDP extension and using the information to retrieve CRLs. Support for CRLDP is optional and [RFC3280] compliant implementations need not populate the CRLDP extension. However, implementers of path-building and validation modules are strongly encouraged to support CRLDPs. At a minimum, developers are encouraged to consider supporting the LDAP and HTTP transports; this will provide for interoperability across a wide range of existing PKIs.

\subsection{Data Obtained via Application Protocol}

Many application protocols, such as SSL/TLS and S/MIME, allow one party to provide certificates and CRLs to another. Data provided in this method is generally very valuable to path-building software (will provide direction toward valid paths), and should be stored and used accordingly. Note: self-signed certificates obtained via application protocol are not trustworthy; implementations should only consider the relying party's trust anchors when building paths.

\subsection{Proprietary Mechanisms}

Some certificate issuing systems and certificate processing systems may utilize proprietary retrieval mechanisms, such as network mapped drives, databases, or other methods that are not directly referenced via the IETF standards. Certificate processing systems may wish to support other proprietary mechanisms, but should only do so in addition to supporting standard retrieval mechanisms such as LDAP, AIA, and CRLDP (unless functioning in a closed environment).

7. Improving Retrieval Performance

Retrieval performance can be improved through a few different mechanisms, including the use of caches and setting a specific retrieval order. This section discusses a few methods by which the performance of a certificate processing system may be improved during the retrieval of PKI objects. Certificate processing systems that are consistently very slow during processing will be disliked by users and will be slow to be adopted into organizations. Certificate processing systems are encouraged to do whatever possible to reduce the delays associated with requesting and retrieving data from external sources. 


\subsection{Caching}

Certificate processing systems operating in a non-hierarchical PKI will often need to retrieve certificates and certificate revocation lists (CRLS) from a source outside the application protocol. Typically, these objects are retrieved from an X.500 or LDAP repository, an Internet URI [RFC3986], or some other non-local source. Due to the delays associated with establishing connections as well as network transfers, certificate processing systems ought to be as efficient as possible when retrieving data from external sources. Perhaps the best way to improve retrieval efficiency is by using a caching mechanism. Certificate processing systems can cache data retrieved from external sources for some period of time, but not to exceed the useful period of the data (i.e., an expired certificate need not be cached). Although this comes at a cost of increased memory/disk consumption by the system, the cost and performance benefit of reducing network transmissions is great. Also, CRLs are often issued and available in advance of the nextupdate date in the CRL. Implementations may wish to obtain these "fresher" CRLs before the nextupdate date has passed.

There are a number of different ways in which caching can be implemented; the specifics of these methods can be used as distinguishing characteristics between certificate processing systems. However, some things that implementers may wish to consider when developing caching systems are as follows:

- If PKI objects are cached, the certification path-building mechanism should be able to examine and retrieve from the cache during path building. This will allow the certificate processing system to find or eliminate one or more paths quickly without requiring external contact with a directory or other retrieval mechanism.

- Sharing caches between multiple users (via a local area network or LAN) may be useful if many users in one organization consistently perform PKI operations with another organization.

- Caching not only PKI objects (such as certificates and CRLs) but also relationships between PKI objects (storing a link between a certificate and the issuer's certificate) may be useful. This linking may not always lead to the most correct or best relationship, but could represent a linking that worked in another scenario.

- Previously built paths and partial paths are quite useful to cache, because they will provide information on previous successes or failures. Additionally, if the cache is safe from 
unauthorized modifications, caching validation and signature checking status for certificates, CRLs, and paths can also be stored.

\subsection{Retrieval Order}

To optimize efficiency, certificate processing systems are encouraged to also consider the order in which different PKI objects are retrieved, as well as the mechanism from which they are retrieved. If caching is utilized, the caches can be consulted for PKI objects before attempting other retrieval mechanisms. If multiple caches are present (such as local disk and network), the caches can be consulted in the order in which they can be expected to return their result from fastest to slowest. For example, if a certificate processing system wishes to retrieve a certificate with a particular subject DN, the system might first consult the local cache, then the network cache, and then attempt directory retrieval. The specifics of the types of retrieval mechanisms and their relative costs are left to the implementer.

In addition to ordering retrieval mechanisms, the certificate processing system ought to order the relative merits of the different external sources from which a PKI object can be retrieved. If the AIA is present within a certificate, with a URI [RFC3986] for the issuer's certificate, the certificate processing system (if able) may wish to attempt to retrieve the certificate first from local cache and then by using that URI (because it is expected to point directly to the desired certificate) before attempting to retrieve the certificates that may exist within a directory.

If a directory is being consulted, it may be desirable to retrieve attributes in a particular order. A highly cross-certified PKI structure will lead to multiple possibilities for certification paths, which may mean multiple validation attempts before a successful path is retrieved. Therefore, cACertificate and usercertificate (which typically contain certificates from within the same 'realm') could be consulted before attempting to retrieve the crosscertificatepair values for an entry. Alternately, all three attributes could be retrieved in one query, but cross-certificates then tagged as such and used only after exhausting the possibilities from the cACertificate attribute. The best approach will depend on the nature of the application and PKI environment.

\subsection{Parallel Fetching and Prefetching}

Much of this document has focused on a path-building algorithm that minimizes the performance impact of network retrievals, by preventing those retrievals and utilization of caches. Another way to improve 
performance would be to allow network retrievals to be performed in advance (prefetching) or at the same time that other operations are performed (parallel fetching). For example, if an email application receives a signed email message, it could download the required certificates and CRLs prior to the recipient viewing (or attempting to verify) the message. Implementations that provide the capability of parallel fetching and/or prefetching, along with a robust cache, can lead to greatly improved performance or user experience.

8. Security Considerations

\subsection{General Considerations for Building a Certification Path}

Although certification path building deals directly with security relevant PKI data, the PKI data itself needs no special handling because its integrity is secured with the digital signature applied to it. The only exception to this is the appropriate protection of the trust anchor public keys. These are to be kept safe and obtained out of band (e.g., not from an electronic mail message or a directory) with respect to the path-building module.

The greatest security risks associated with this document revolve around performing certification path validation while certification paths are built. It is therefore noted here that fully implemented certification path validation in accordance with [RFC3280] and [X.509] is required in order for certification path building, certification path validation, and the certificate using application to be properly secured. All of the Security Considerations listed in Section 9 of [RFC3280] apply equally here.

In addition, as with any application that consumes data from potentially untrusted network locations, certification path-building components should be carefully implemented so as to reduce or eliminate the possibility of network based exploits. For example, a poorly implemented path-building module may not check the length of the CRLDP URI [RFC3986] before using the C language strcpy() function to place the address in a 1024 byte buffer. A hacker could use such a flaw to create a buffer overflow exploit by encoding malicious assembly code into the CRLDP of a certificate and then use the certificate to attempt an authentication. Such an attack could yield system level control to the attacker and expose the sensitive data the PKI was meant to protect.

Path building may be used to mount a denial of service (DoS) attack. This might occur if multiple simple requests could be performed that cause a server to perform a number of path developments, each taking time and resources from the server. Servers can help avoid this by limiting the resources they are willing to devote to path building, 
and being able to further limit those resources when the load is heavy. Standard DOS protections such as systems that identify and block attackers can also be useful.

A DOS attack can be also created by presenting spurious CA certificates containing very large public keys. When the system attempts to use the large public key to verify the digital signature on additional certificates, a long processing delay may occur. This can be mitigated by either of two strategies. The first strategy is to perform signature verifications only after a complete path is built, starting from the trust anchor. This will eliminate the spurious CA certificate from consideration before the large public key is used. The second strategy is to recognize and simply reject keys longer than a certain size.

A similar Dos attack can occur with very large public keys in end entity certificates. If a system uses the public key in a certificate before building and validating that certificate's certification path, long processing delays may occur. To mitigate this threat, the public key in an end entity certificate should not be used for any purpose until a complete certification path for that certificate is built and validated.

\subsection{Specific Considerations for Building Revocation Signer} Certification Paths

If the CRL Signer certificate (and certification path) is not identical to the Certification Authority certificate (and certification path), special care should be exercised when building the CRL Signer certification path.

If special consideration is not given to building a CRL Signer certification path, that path could be constructed such that it terminates with a different root or through a different certification path to the same root. If this behavior is not prevented, the relying party may end up checking the wrong revocation data, or even maliciously substituted data, resulting in denial of service or security breach.

For example, suppose the following certification path is built for E and is valid for an example "high assurance" policy.

$$
\mathrm{A}->\mathrm{B}->\mathrm{C}->\mathrm{E}
$$

When the building/validation routine attempts to verify that $\mathrm{E}$ is not revoked, $\mathrm{C}$ is referred to as the Certification Authority certificate. The path builder finds that the CRL for checking the revocation status of $\mathrm{E}$ is issued by $\mathrm{C} 2$; a certificate with the subject name "C", 
but with a different key than the key that was used to sign E. C2 is referred to as the CRL Signer. An unrestrictive certification path builder might then build a path such as the following for the CRL Signer C2 certificate:

$$
X->Y->Z->C 2
$$

If a path such as the one above is permitted, nothing can be concluded about the revocation status of $\mathrm{E}$ since $\mathrm{C} 2$ is a different $\mathrm{CA}$ from C.

Fortunately, preventing this security problem is not difficult and the solution also makes building CRL Signer certification paths very efficient. In the event the CRL Signer certificate is identical to the Certification Authority certificate, the Certification Authority certification path should be used to verify the CRL; no additional path building is required. If the CRL Signer certificate is not identical to the Certification Authority certificate, a second path should be built for the CRL Signer certificate in exactly the same fashion as for any certificate, but with the following additional guidelines:

1. Trust Anchor: The CRL Signer's certification path should start with the same trust anchor as the Certification Authority's certification path. Any trust anchor certificate with a subject DN matching that of the Certification Authority's trust anchor should be considered acceptable though lower in priority than the one with a matching public key and subject DN. While different trust anchor public keys are acceptable at the beginning of the CRL signer's certification path and the Certification Authority's certification path, both keys must be trusted by the relying party per the recommendations in section 8.1 .

2. CA Name Matching: The subject DNs for all CA certificates in the two certification paths should match on a one-to-one basis (ignoring self-issued certificates) for the entire length of the shorter of the two paths.

3. CRL Signer Certification Path Length: The length of the CRL Signer certification path (ignoring self-issued certificates) should be equal to or less than the length of the Certification Authority certification path plus (+) one. This allows a given Certification Authority to issue a certificate to a delegated/subordinate CRL Signer. The latter configuration represents the maximum certification path length for a CRL Signer certificate. 
The reasoning behind the first guideline is readily apparent. Lacking this and the second guideline, any trusted CA could issue CRLs for any other CA, even if the PKIs are not related in any fashion. For example, one company could revoke certificates issued by another company if the relying party trusted the trust anchors from both companies. The two guidelines also prevent erroneous CRL checks since Global uniqueness of names is not guaranteed.

The second guideline prevents roaming certification paths such as the previously described example CRL Signer certification path for $\mathrm{A}->\mathrm{B}->\mathrm{C}->\mathrm{E}$. It is especially important that the "ignoring selfissued certificates" is implemented properly. Self-issued certificates are cast out of the one-to-one name comparison in order to allow for key rollover. The path-building algorithm may be optimized to only consider certificates with the acceptable subject DN for the given point in the CRL Signer certification path while building the path.

The third and final guideline ensures that the CRL used is the intended one. Without a restriction on the length of the CRL Signer certification path, the path could roam uncontrolled into another domain and still meet the first two guidelines. For example, again using the path $\mathrm{A}->\mathrm{B}->\mathrm{C}->\mathrm{E}$, the Certification Authority $\mathrm{C}$, and a CRL Signer C2, a CRL Signer certification path such as the following could pass the first two guidelines:

$$
\mathrm{A}->\mathrm{B}->\mathrm{C}->\mathrm{D}->\mathrm{X}->\mathrm{Y}->\mathrm{R} \text { ogueCA }->\mathrm{C} 2
$$

In the preceding example, the trust anchor is identical for both paths and the one-to-one name matching test passes for $\mathrm{A}->\mathrm{B}->\mathrm{C}$. However, accepting such a path has obvious security consequences, so the third guideline is used to prevent this situation. Applying the second and third guideline to the certification path above, the path builder could have immediately detected this path was not acceptable (prior to building it) by examining the issuer DN in C2. Given the length and name guidelines, the path builder could detect that "RogueCA" is not in the set of possible names by comparing it to the set of possible CRL Signer issuer DNs, specifically, A, B, or C.

Similar consideration should be given when building the path for the OCSP Responder certificate when the CA is the OCSP Response Signer or the CA has delegated the OCSP Response signing to another entity. 


\section{Acknowledgements}

The authors extend their appreciation to David Lemire for his efforts coauthoring "Managing Interoperability in Non-Hierarchical Public Key Infrastructures" from which material was borrowed heavily for use in the introductory sections.

This document has also greatly benefited from the review and additional technical insight provided by Dr. Santosh Chokhani, Carl Wallace, Denis Pinkas, Steve Hanna, Alice Sturgeon, Russ Housley, and Tim Polk.

\section{Normative References}

[RFC3280] Housley, R., Polk, W., Ford, W., and D. Solo, "Internet X.509 Public Key Infrastructure Certificate and Certificate Revocation List (CRL) Profile", RFC 3280, April 2002 .

11. Informative References

[MINHPKIS] Hesse, P., and D. Lemire, "Managing Interoperability in Non-Hierarchical Public Key Infrastructures", 2002 Conference Proceedings of the Internet Society Network and Distributed System Security Symposium, February 2002.

[RFC1777] Yeong, W., Howes, T., and S. Kille, "Lightweight Directory Access Protocol", RFC 1777, March 1995.

[RFC2560] Myers, M., Ankney, R., Malpani, A., Galperin, S., and C. Adams, "X.509 Internet Public Key Infrastructure Online Certificate Status Protocol - OCSP", RFC 2560, June 1999.

[RFC2587] Boeyen, S., Howes, T., and P. Richard, "Internet X.509 Public Key Infrastructure LDAPv2 Schema", RFC 2587, June 1999 .

[RFC3377] Hodges, J. and R. Morgan, "Lightweight Directory Access Protocol (v3): Technical Specification", RFC 3377, September 2002 .

[RFC3820] Tuecke, S., Welch, V., Engert, D., Pearlman, L., and M. Thompson, "Internet X.509 Public Key Infrastructure (PKI) Proxy Certificate Profile", RFC 3820, June 2004.

[RFC3986] Berners-Lee, T., Fielding, R., and L. Masinter, "Uniform Resource Identifier (URI): Generic Syntax", STD 66, RFC 3986, January 2005. 
[X.501] ITU-T Recommendation X.501: Information Technology - Open Systems Interconnection - The Directory: Models, 1993.

[X.509] ITU-T Recommendation X.509 (2000 E): Information Technology - Open Systems Interconnection - The Directory: Authentication Framework, March 2000.

[PKIXALGS] Bassham, L., Polk, W. and R. Housley, "Algorithms and Identifiers for the Internet X.509 Public Key Infrastructure Certificate and Certificate Revocation Lists (CRL) Profile", RFC 3279, April 2002 .

[CERTSTORE] P. Gutmann, "Internet X.509 Public Key Infrastructure Operational Protocols: Certificate Store Access via HTTP", Work in Progress, August 2004. 


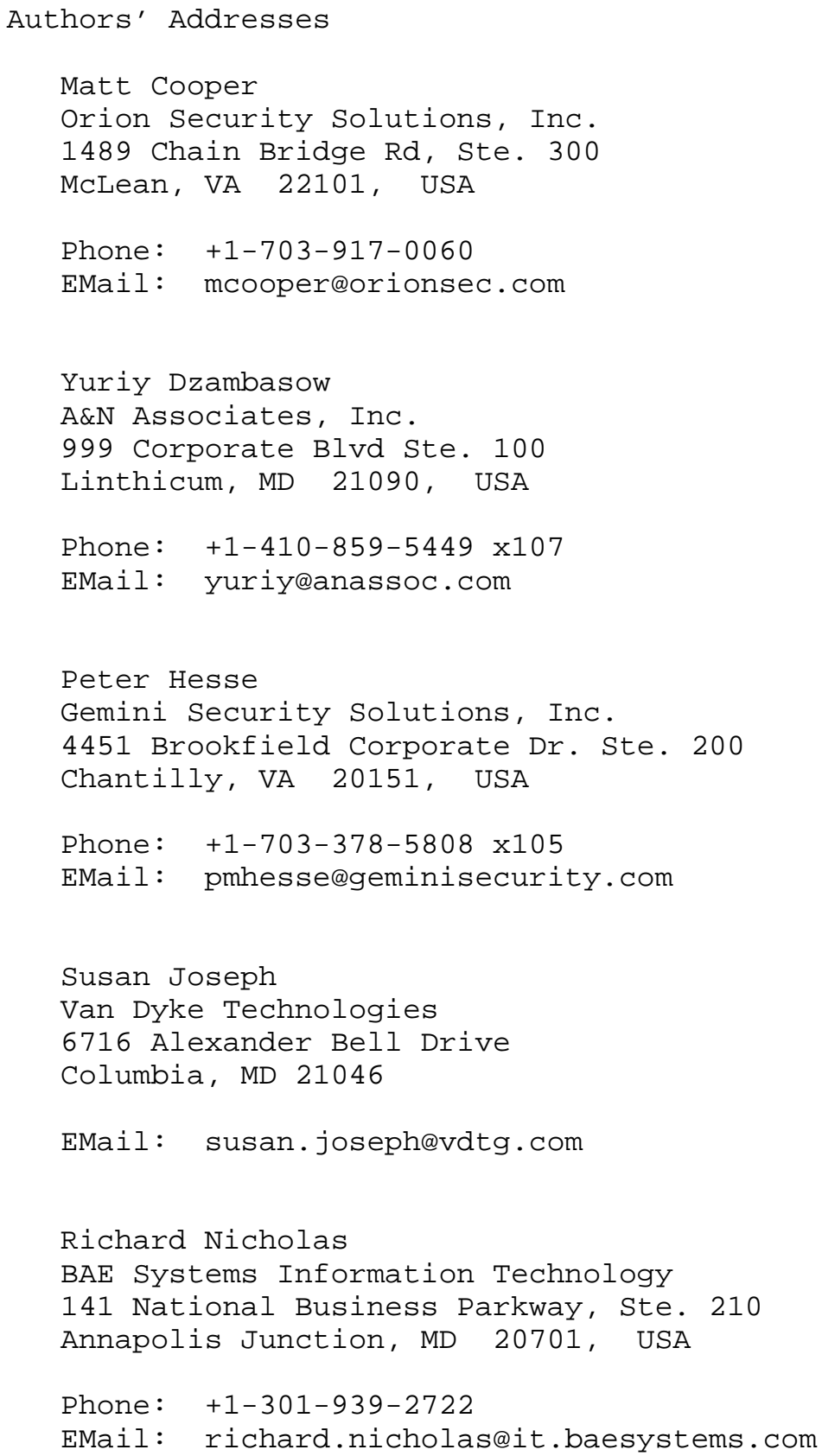


Full Copyright statement

Copyright (C) The Internet Society (2005).

This document is subject to the rights, licenses and restrictions contained in BCP 78, and except as set forth therein, the authors retain all their rights.

This document and the information contained herein are provided on an "AS IS" basis and THE CONTRIBUTOR, THE ORGANIZATION HE/SHE REPRESENTS OR IS SPONSORED BY (IF ANY), THE INTERNET SOCIETY AND THE INTERNET ENGINEERING TASK FORCE DISCLAIM ALL WARRANTIES, EXPRESS OR IMPLIED, INCLUDING BUT NOT LIMITED TO ANY WARRANTY THAT THE USE OF THE INFORMATION HEREIN WILL NOT INFRINGE ANY RIGHTS OR ANY IMPLIED WARRANTIES OF MERCHANTABILITY OR FITNESS FOR A PARTICULAR PURPOSE.

Intellectual Property

The IETF takes no position regarding the validity or scope of any Intellectual Property Rights or other rights that might be claimed to pertain to the implementation or use of the technology described in this document or the extent to which any license under such rights might or might not be available; nor does it represent that it has made any independent effort to identify any such rights. Information on the procedures with respect to rights in RFC documents can be found in BCP 78 and BCP 79 .

Copies of IPR disclosures made to the IETF Secretariat and any assurances of licenses to be made available, or the result of an attempt made to obtain a general license or permission for the use of such proprietary rights by implementers or users of this specification can be obtained from the IETF on-line IPR repository at http://www.ietf.org/ipr.

The IETF invites any interested party to bring to its attention any copyrights, patents or patent applications, or other proprietary rights that may cover technology that may be required to implement this standard. Please address the information to the IETF at ietfipreietf.org.

Acknowledgement

Funding for the RFC Editor function is currently provided by the Internet Society. 